\title{
MOTIVIC COHOMOLOGY, LOCALIZED CHERN CLASSES, AND LOCAL TERMS
}

\author{
MARTIN OLSSON
}

\begin{abstract}
Let $c: C \rightarrow X \times X$ be a correspondence with $C$ and $X$ quasi-projective schemes over an algebraically closed field $k$. We show that if $u_{\ell}: c_{1}^{*} \mathbb{Q}_{\ell} \rightarrow c_{2}^{!} \mathbb{Q}_{\ell}$ is an action defined by the localized Chern classes of a $c_{2}$-perfect complex of vector bundles on $C$, where $\ell$ is a prime invertible in $k$, then the local terms of $u_{\ell}$ are given by the class of an algebraic cycle independent of $\ell$. We also prove some related results for quasi-finite correspondences. The proofs are based on the work of Cisinski and Deglise on triangulated categories of motives.
\end{abstract}

\section{Contents}

1. Introduction 1

2. Motivic categories and the six operations 3

3. Chern classes 6

4. Local Chern classes 9

$\begin{array}{ll}\text { 5. Local terms for motivic actions } & 11\end{array}$

$\begin{array}{ll}\text { 6. Beilinson motives } & 13\end{array}$

\begin{tabular}{lll}
\hline 7. & Application: local terms for actions given by localized Chern classes & 19
\end{tabular}

\begin{tabular}{lll}
\hline 8. Application: quasi-finite morphisms and correspondences & 19
\end{tabular}

$\begin{array}{ll}\text { References } & 26\end{array}$

\section{INTRODUCTION}

The motivation for this work comes from our study of local terms arising from actions of correspondences defined by local Chern classes of complexes of vector bundles in [16. The purpose of the present paper is to elucidate the motivic nature of these local terms using the machinery developed by Cisinski and Deglise in [4].

The basic problem we wish to address is the following. Fix an algebraically closed field $k$ of characteristic $p$ (possibly 0 ), and let $\mathscr{S}$ denote the category of finite type separated $k$-schemes. Let $c: C \rightarrow X \times X$ be a correspondence with $C, X \in \mathscr{S}$. A $c_{2}$-perfect complex $E$ on $C$ defines for any prime $\ell$ invertible in $k$ an action $u_{\ell}: c_{1}^{*} \mathbb{Q}_{\ell} \rightarrow c_{2}^{!} \mathbb{Q}_{\ell}$, and therefore by the general machinery of SGA 5 a class $\operatorname{Tr}\left(u_{\ell}\right) \in H^{0}\left(\operatorname{Fix}(c), \Omega_{\mathrm{Fix}(c)}\right)$, where $\operatorname{Fix}(c)$ denotes the scheme of fixed points $\operatorname{Fix}(c):=C \times_{c, X \times X, \Delta_{X}} X$ and $\Omega_{\mathrm{Fix}(c)}$ is the $\ell$-adic dualizing complex (see [12, III, $\S 4]$ for further discussion). Recall from loc. cit. that for any proper connected component 
$Z \subset \operatorname{Fix}(c)$ the local term of $u_{\ell}$ is given by the proper pushforward of the restriction of $\operatorname{Tr}\left(u_{\ell}\right)$ to $Z$, and consequently in good situations can be used via the Grothendieck-Lefschetz trace formula [12, III, 4.7] to calculate the trace of the induced action of $u_{\ell}$ on global cohomology.

On the other hand, $H^{0}\left(\operatorname{Fix}(c), \Omega_{\mathrm{Fix}(c)}\right)$ is the $\ell$-adic Borel-Moore homology of $\operatorname{Fix}(c)$ and there is a cycle class map

$$
\mathrm{cl}_{\ell}: A_{0}(\operatorname{Fix}(c)) \rightarrow H^{0}\left(\operatorname{Fix}(c), \Omega_{\mathrm{Fix}(c)}\right),
$$

where $A_{0}(\operatorname{Fix}(c))$ denotes the group of 0 -cycles on $\operatorname{Fix}(c)$ modulo rational equivalence.

The main result about local terms in this paper is the following:

Theorem 1.1 (Theorem 7.4). There exists a zero-cycle $\Sigma \in A_{0}(\operatorname{Fix}(c))_{\mathbb{Q}}$ such that for any prime $\ell$ invertible in $k$ the class $\operatorname{Tr}\left(u_{\ell}\right)$ is equal to $\mathrm{cl}_{\ell}(\Sigma)$.

As we explain, this theorem is a fairly formal consequence of a suitable theory of derived categories of motives and six operations for such categories. The fact that such a theory exists is due to Cisinski and Deglise [4. They developed a notion of triangulated motivic categories with a six operations formalism realizing a vision of Beilinson. Roughly speaking such a category is a fibered category $\mathscr{M}$ over $\mathscr{S}$ such that for every $X \in \mathscr{S}$ the fiber $\mathscr{M}(X)$ is a monoidal triangulated category and for every morphism $f: X \rightarrow Y$ in $\mathscr{S}$ we have functors

$$
f_{!}, f_{*}: \mathscr{M}(X) \rightarrow \mathscr{M}(Y), \quad f^{*}, f^{!}: \mathscr{M}(Y) \rightarrow \mathscr{M}(X)
$$

satisfying the usual properties. In addition there should be a suitable notion of Chern classes. Already in this context we can define localized Chern classes of complexes of vector bundles as well as analogous of the classes $\operatorname{Tr}\left(u_{\ell}\right)$, which are functorial in $\mathscr{M}$. In particular, we can consider the category $\mathscr{M}_{B}$ of Beilinson motives defined in [4, §14]. In this case, the $\mathscr{M}_{B^{-}}$ version of $H^{0}\left(\operatorname{Fix}(c), \Omega_{\mathrm{Fix}(c)}\right)$ is simply the group $A_{0}(\operatorname{Fix}(c))_{\mathbb{Q}}$ and the $\mathscr{M}_{B^{-}}$version of $\operatorname{Tr}\left(u_{\ell}\right)$ is the cycle appearing in 1.1. Using [5], we then get 1.1 by passing to étale realizations.

The proof of 1.1 can essentially be phrased as saying that actions arising from $c_{2}$-perfect complexes are motivic. In general it seems a difficult question to prove that a given action of a correspondence is motivic. There is one other case, however, where one can fairly easily detect if an action is motivic. Namely, for a quasi-finite morphism $f: Y \rightarrow X$ there is a natural necessary condition for a section $u_{\ell} \in H^{0}\left(Y, f^{!} \mathbb{Q}_{\ell}\right)$ to be the étale realization of a morphism $u: 1_{Y} \rightarrow f^{!} 1_{X}$ in the triangulated category of Beilinson motives over $Y$. In theorem 8.2 we show that this condition is also sufficient. This also has global consequences. In particular, a special case of theorem 8.18 is the following:

Theorem 1.2. Let $k$ be an algebraically closed field and let $X / k$ be a separated DeligneMumford stack. Let $f: X \rightarrow X$ be a finite morphism (as a morphism of stacks). Then the alternating sum of traces

$$
\sum_{i}(-1)^{i} \operatorname{tr}\left(f^{*} \mid H^{i}\left(X, \mathbb{Q}_{\ell}\right)\right)
$$

is in $\mathbb{Q}$ and independent of $\ell$.

Remark 1.3. Since the trace appearing 1.2 is in $\mathbb{Z}_{\ell}$ it follows that the alternating sum of traces is in $\mathbb{Z}[1 / p]$, where $p$ is the characteristic of $k$. In fact, notice that since $R \Gamma\left(X, \mathbb{Z}_{\ell}\right)$ is a perfect complex we can define $\operatorname{tr}\left(u^{*} \mid R \Gamma\left(X, \mathbb{Z}_{\ell}\right)\right) \in \mathbb{Z}_{\ell}$, which by the above is an element of 
$\mathbb{Z}[1 / p]$ which reduces $\bmod \ell$ to $\operatorname{tr}\left(u^{*} \mid R \Gamma\left(X, \mathbb{F}_{\ell}\right)\right)$, thereby yielding $\ell$-independence for $\bmod \ell$ traces as well.

Remark 1.4. One might hope more generally to use the techniques of this paper to study motivic local terms with $\mathbb{Z}$ coefficients to obtain cycles in $A_{0}(\operatorname{Fix}(c))$ before tensoring with $\mathbb{Q}$. However, the theory at present seems restricted to $\mathbb{Q}$-coefficients as the six operations on a suitable triangulated category of motives is not known to exist integrally. Work in preparation by Cisinski and Deglise on integral motives may, however, lead to integral results.

Remark 1.5. In this paper we discuss étale cohomology and local terms defined in the étale theory. However, with a suitable theory of $p$-adic local terms and $p$-adic realization functors one would also get rationality of $p$-adic local terms and compatility with the étale local terms.

Remark 1.6. Theorem 1.2 has also been obtained by Bondarko using variant motivic methods [3, Discussion following 8.4.1].

Remark 1.7. Many of the foundational results obtained in this paper hold not just over a field but over more general base schemes and we develop the theory in greater generality. For the applications to local terms, however, it suffices to work over an algebraically closed field.

1.8. Acknowledgements. The author is grateful to Doosung Park for suggesting that the work of Cisinski and Deglise should imply 1.1, and for comments of Cisinski and Deglise on a preliminary draft. The author was partially supported by NSF CAREER grant DMS-0748718 and NSF grant DMS-1303173.

\section{Motivic CATEgories AND THE SiX OPERATIONS}

Let $B$ be a regular separated scheme of finite dimension, and let $\mathscr{S}$ denote the category of finite type separated $B$-schemes.

2.1. Recall from [4, Section 1] that a triangulated premotivic category $\mathscr{M}$ is a fibered category over $\mathscr{S}$ satisfying the following five conditions (a good summary is given in [5, A.1.1]):

(PM1) For every $S \in \mathscr{S}$ the fiber category $\mathscr{M}(S)$ is a well-generated (in the sense of [15]) triangulated category with a closed monoidal structure.

(PM2) For every morphism $f: X \rightarrow Y$ in $\mathscr{S}$ the functor (well-defined up to unique isomorphism)

$$
f^{*}: \mathscr{M}(Y) \rightarrow \mathscr{M}(X)
$$

is triangulated, monoidal, and admits a right adjoint $f_{*}$.

(PM3) For every smooth morphism $f: X \rightarrow Y$ in $\mathscr{S}$ the functor $f^{*}: \mathscr{M}(Y) \rightarrow \mathscr{M}(X)$ admits a left adjoint $f_{\sharp}$.

(PM4) For every cartesian square with $p$ smooth

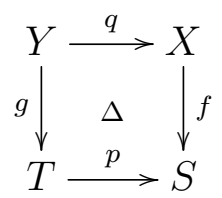

there is a canonical isomorphism of functors

$$
\operatorname{Ex}\left(\Delta_{\sharp}^{*}\right): q_{\sharp} g^{*} \simeq f^{*} p_{\sharp} .
$$


(PM5) For every smooth morphism $p: T \rightarrow S, M \in \mathscr{M}(T)$, and $N \in \mathscr{M}(S)$ then there is a canonical isomorphism

$$
\operatorname{Ex}\left(p_{\sharp}^{*}, \otimes\right): p_{\sharp}\left(M \otimes_{T} p^{*} N\right) \simeq p_{\sharp}(M) \otimes_{S} N .
$$

Remark 2.2. Note that for any category $\mathscr{S}$ we can talk about a triangulated fibered category over $\mathscr{S}$. By this we mean a fibered category $p: \mathscr{M} \rightarrow \mathscr{S}$ satisfying axioms (PM1)-(PM3) and (PM4).

2.3. For every $X \in \mathscr{S}$, the monoidal structure on $\mathscr{M}(X)$ gives a unit object $1_{X} \in \mathscr{M}(X)$. For a smooth morphism $f: X \rightarrow S$ in $\mathscr{S}$ define $M_{S}(X) \in \mathscr{M}(S)$ to be $f_{\sharp}\left(1_{X}\right)$. Because the pullback functor $f^{*}$ is monoidal we have $f^{*} 1_{S}=1_{X}$ and therefore by adjunction a morphism

$$
M_{S}(X)=f_{\sharp} f^{*} 1_{S} \stackrel{f_{\sharp} f^{*} \rightarrow \mathrm{id}}{\longrightarrow} 1_{S},
$$

which we denote by $a_{X / S}$.

A Tate motive for $\mathscr{M}$ is a cartesian section $\tau: \mathscr{S} \rightarrow \mathscr{M}$ with $\tau(S)$ fitting into a distringuished triangle

$$
\tau(S) \longrightarrow M_{S}\left(\mathbb{P}_{S}^{1}\right) \stackrel{a_{\mathbb{P}_{S}^{1}} / S}{\longrightarrow} 1_{S} \longrightarrow \tau(S)
$$

functorial in $S$. We usually write just $1_{S}(1)$ for $\tau(S)$.

2.4. We can consider various other natural axioms on a triangulated premotivic category with a Tate object:

(Homotopy axiom) For every $S \in \mathscr{S}$ the map

$$
a_{\mathbb{A}_{S}^{1} / S}: M_{S}\left(\mathbb{A}_{S}^{1}\right) \rightarrow 1_{S}
$$

is an isomorphism.

(Stability property) The Tate motive $1_{S}(1)$ is $\otimes$-invertible. In this case we get motives $1_{S}(n)$ for all $n \in \mathbb{Z}$.

2.5. Given a triangulated premotivic category $\mathscr{M}$ with a Tate motive satisfying the stability property we define motivic cohomology, a bigraded cohomology theory on $\mathscr{S}$, by

$$
H_{\mathscr{M}}^{i, n}(S):=\operatorname{Hom}_{\mathscr{M}(S)}\left(1_{S}, 1_{S}(n)[i]\right) .
$$

2.6. A morphism between two triangulated premotivic categories $\mathscr{M}$ and $\mathscr{M}^{\prime}$ is a cartesian functor $\varphi^{*}: \mathscr{M} \rightarrow \mathscr{M}^{\prime}$ such that the following hold:

(i) For every $S \in \mathscr{S}$ the functor $\varphi_{S}^{*}: \mathscr{M}(S) \rightarrow \mathscr{M}\left(S^{\prime}\right)$ is a triangulated monoidal functor which admits a right adjoint $\varphi_{S *}$.

(ii) For every smooth morphism $p: T \rightarrow S$ in $\mathscr{S}$ there is a canonical isomorphism

$$
\operatorname{Ex}\left(p_{\sharp}, \varphi^{*}\right): p_{\sharp} \varphi_{T}^{*} \rightarrow \varphi_{S}^{*} p_{\sharp} .
$$

In fact triangulated premotivic categories form a 2-category in which the above morphisms are the 1-morphisms, and 2-morphisms are given by morphisms of cartesian functors $\epsilon: \varphi^{*} \rightarrow \psi^{*}$ compatible with the structures in (i) and (ii).

Remark 2.7. Similarly we can consider the 2-category of triangulated fibered categories over any base category $\mathscr{S}$. 
2.8. Let $\mathscr{S}$ be as above, and let $\operatorname{Ar}(\mathscr{S})$ be the category of morphisms in $\mathscr{S}$. We have two functors

$$
s, t: \operatorname{Ar}(\mathscr{S}) \rightarrow \mathscr{S}
$$

given by the source and target respectively. For a triangulated premotivic category $\mathscr{M}$ over $\mathscr{S}$ let $\mathscr{M}^{s}\left(\right.$ resp. $\left.\mathscr{M}^{t}\right)$ denote $s^{*} \mathscr{M}\left(\right.$ resp. $\left.t^{*} \mathscr{M}\right)$, a triangulated fibered category over $\operatorname{Ar}(\mathscr{S})$.

A six functor formalism for $\mathscr{M}$ consists of the following data:

(1) 2-functors $f \mapsto f_{*}$ and $f \mapsto f_{\text {! from }} \mathscr{M}^{s} \rightarrow \mathscr{M}^{t}$ and $f \mapsto f^{*}$ and $f \mapsto f^{!}$from $\mathscr{M}^{t}$ to $\mathscr{M}^{s}$ such that for every $f: X \rightarrow Y \in \operatorname{Ar}(\mathscr{S})$ the functors $f_{*}$ and $f^{*}$ are as previously defined, and $f_{!}$is left adjoint to $f$ !

(2) There exists a morphism of 2-functors $\alpha: f_{!} \rightarrow f_{*}$ which is an isomorphism if $f$ is proper.

(3) For any smooth morphism $f: X \rightarrow S$ in $\mathscr{S}$ of relative dimension $d$ there are isomorphisms $\mathfrak{p}_{f}: f_{\sharp} \rightarrow f_{!}(d)[2 d]$ and $\mathfrak{p}_{f}^{\prime}: f^{*} \simeq f^{!}(-d)[-2 d]$. These are given by isomorphisms of 2 -functors on the category of smooth morphisms of relative dimension $d$.

(4) For every cartesian square

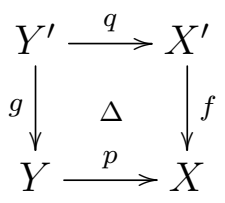

there are natural isomorphisms of functors

$$
\begin{aligned}
p^{*} f_{!} & \simeq g_{!} q^{*}, \\
g_{*} q^{!} & \simeq p^{!} f_{*} .
\end{aligned}
$$

(5) For every $f: Y \rightarrow X$ there are natural isomorphisms

$$
\begin{gathered}
\operatorname{Ex}\left(f_{!}^{*}, \otimes\right):\left(f_{!} K\right) \otimes_{X} L \simeq f_{!}\left(K \otimes_{Y} f^{*} L\right), \\
\operatorname{Hom}_{X}\left(f_{!} L, K\right) \simeq f_{*} \operatorname{Hom}_{Y}\left(L, f^{!} K\right),
\end{gathered}
$$

and

$$
f^{!} \operatorname{Hom}_{X}(L, M) \simeq \operatorname{Hom}_{Y}\left(f^{*} L, f_{!} M\right) .
$$

(Loc) Let $X \in \mathscr{S}$ be an object, $i: Z \hookrightarrow X$ a closed imbedding, and let $j: U \hookrightarrow X$ be the complementary open set. Then there exists a map of functors $\partial: i_{*} i^{*} \rightarrow j_{!} !^{!}[1]$ such for every $F \in M(X)$ the induced triangle

$$
j ! j ! F \longrightarrow F \longrightarrow i_{*} i^{*} F \stackrel{\partial}{\longrightarrow} j_{!} j^{!} F[1]
$$

is distinguished, where the first two maps are those induced by adjunction.

Finally Deglise and Cisinski consider purity and duality properties:

(Relative Purity) For a closed immersion $i: Z \hookrightarrow X$ of smooth separated $B$-schemes there is a canonical isomorphism

$$
1_{Z}(-c)[-2 c] \simeq i^{!}\left(1_{X}\right)
$$

where $c$ is the codimension of $Z$ in $X$. 
(Duality) For $X \in \mathscr{S}$ with structure morphism $f: X \rightarrow B$ we write $\Omega_{X}^{\mathscr{M}}$ (or just $\Omega_{X}$ if no confusion seems likely to arise) for $f^{!} 1_{B} \in \mathscr{M}(X)$. Define $D_{X}: \mathscr{M}(X)^{\text {op }} \rightarrow \mathscr{M}(X)$ to be the functor $M \mapsto \operatorname{Hom}_{X}\left(M, \Omega_{X}^{\mathscr{M}}\right)$.

(a) For every $M \in \mathscr{M}(X)$ the natural map

$$
M \rightarrow D_{X}\left(D_{X}(M)\right)
$$

is an isomorphism.

(b) For every $X$ and $M, N \in \mathscr{M}(X)$ we have a canonical isomorphism

$$
D_{X}\left(M \otimes D_{X}(N)\right) \simeq \operatorname{Hom}_{X}(M, N) \text {. }
$$

(c) For every $f: Y \rightarrow X$ in $\mathscr{S}, M \in \mathscr{M}(X)$, and $N \in \mathscr{M}(Y)$ we have natural isomorphisms

$$
\begin{aligned}
D_{Y}\left(f^{*}(M)\right) & \simeq f^{!}\left(D_{X}(M)\right), \\
f^{*} D_{X}((M)) & \simeq D_{Y}\left(f^{!}(M)\right), \\
D_{X}\left(f_{!}(N)\right) & \simeq f_{*}\left(D_{Y}(N)\right), \\
f_{!}\left(D_{Y}(N)\right) & \simeq D_{X}\left(f_{*}(N)\right) .
\end{aligned}
$$

We say that a triangulated premotivic category $\mathscr{M}$ is a triangulated motivic category over $\mathscr{S}$ if all of the above conditions hold.

Remark 2.9. This is stronger than what is in [4, 2.4.45] but we will not need their slightly weaker notion.

Remark 2.10. The relative purity property follows from property 2.8 (3), but we state it explicitly for later use.

Remark 2.11. If $R$ is a ring we can also consider a notion of an $R$-linear triangulated motivic category over $\mathscr{S}$. By definition this means that each $\mathscr{M}(X)$ is an $R$-linear symmetric monoidal triangulated category, and that all the above structure respects this $R$-linear structure.

\section{Chern Classes}

As in the previous section let $B$ be a regular separated scheme of finite dimension, and let $\mathscr{S}$ denote the category of finite type separated $B$-schemes.

3.1. Fix an $R$-linear triangulated motivic category $\mathscr{M}$ over $\mathscr{S}$. For $n, m \in \mathbb{Z}$ let

$$
H_{\mathscr{M}}^{n, m}: \mathscr{S}^{\text {op }} \rightarrow \operatorname{Mod}_{R}
$$

be the functor sending $X \in \mathscr{S}$ to $H_{\mathscr{M}}^{n, m}(X):=\operatorname{Hom}_{\mathscr{M}(X)}\left(1_{X}, 1_{X}(m)[n]\right)$.

Let Pic (resp. Vec, $K^{0}$ ) be the functor on $\mathscr{S}$ sending $X$ to the Picard group Pic $(X)$ of $X$ (resp. the set of isomorphism classes of finite rank vector bundles on $X$, the Grothendieck group of vector bundles on $X$ ). A pre-orientation on $\mathscr{M}$ is a morphism of functors

$$
c_{1}: \text { Pic } \rightarrow H_{\mathscr{M}}^{2,1} \text {. }
$$

Let $X \in \mathscr{S}$ be an object smooth over $B$ and let $i: Z \hookrightarrow X$ be a Cartier divisor smooth over $B$. By relative purity, we get a canonical isomorphism

$$
i^{!} 1_{X} \simeq 1_{Z}(-1)[-2] .
$$


Combining this with the adjunction $i_{*} i^{!} \rightarrow \mathrm{id}_{X}$ we get a morphism

$$
H^{0,0}(Z) \rightarrow H^{2,1}(X)
$$

We say that a pre-orientation $c_{1}$ is an orientation if this map sends the identity class in $H^{0,0}(Z)$ to $c_{1}\left(\mathscr{O}_{X}(Z)\right)$ for every such closed imbedding $i: Z \hookrightarrow X$.

For the remainder of this section we fix an orientation $c_{1}$ on $\mathscr{M}$.

3.2. A theory of Chern classes for $\mathscr{M}$ is a collection of morphisms of functors

$$
c_{n}: \operatorname{Vec} \rightarrow H_{\mathscr{M}}^{2 n, n}, \quad n \geq 0
$$

such that the following conditions hold:

(i) $c_{0}$ is the constant 1 and $c_{1}$ is the given orientation.

(ii) (Vanishing) For a vector bundle $E$ on $X$ of rank $r$ we have $c_{i}(E)=0$ for $i>r$.

(iii) (Commutativity) For vector bundles $E$ and $F$ on $X \in \mathscr{S}$ and integers $i, j \in \mathbb{Z}$ we have

$$
c_{i}(E) \cdot c_{j}(F)=c_{j}(F) \cdot c_{i}(E) .
$$

(iv) (Whitney sum) For a short exact sequence of vector bundles on $X \in \mathscr{S}$

$$
0 \rightarrow E^{\prime \prime} \rightarrow E \rightarrow E^{\prime} \rightarrow 0
$$

we have

$$
c_{k}(E)=\sum_{i+j=k} c_{i}\left(E^{\prime \prime}\right) c_{j}\left(E^{\prime}\right) .
$$

Remark 3.3. When $R$ is a $\mathbb{Q}$-algebra, we can define as usual the Chern character which defines a morphism of functors

$$
\operatorname{ch}: K^{0} \rightarrow \prod_{n} H_{\mathscr{M}}^{2 n, n}
$$

as well as Todd classes.

3.4. Assume given a theory of Chern classes for $\mathscr{M}$. The classical computations of cohomology for flag varieties can then be carried out in our cohomology theory as well. Let us briefly recall the statement and construction. For $X \in \mathscr{S}$ define

$$
A_{\mathscr{M}}^{n, m}(X):=H_{\mathscr{M}}^{2 n, m}(X)
$$

and set

$$
A_{\mathscr{M}}^{*, *}(X):=\oplus_{n, m \in \mathbb{Z}} A_{\mathscr{M}}^{n, m}(X) .
$$

Then $A_{\mathscr{M}}^{*, *}(X)$ is a bigraded ring. For $F \in \mathscr{M}(X)$ define

$$
A_{\mathscr{M}}^{n, m}(X, F):=\operatorname{Ext}_{\mathscr{M}(X)}^{2 n}\left(1_{X}, F(m)\right)
$$

and set

$$
A_{\mathscr{M}}^{*, *}(X, F):=\oplus_{n, m} A_{\mathscr{M}}^{n, m}(X, F),
$$

a module over $A_{\mathscr{M}}^{*, *}(X)$. The main case of interest is when $F=1_{X}(m)[2 n]$ for some $n$ and $m$, in which case $A_{\mathscr{M}}^{*, *}(X, F)$ is a free module of rank 1 over $A_{\mathscr{M}}^{*, *}(X)$ with generator in degree $A_{\mathscr{M}}^{-n,-m}(X, F)$. 
3.5. Let $X \in \mathscr{S}$ be a scheme, let $E$ be a vector bundle on $X$. Fix a sequence of integers $\left(p_{1}, \ldots, p_{m}\right)$ and let $p: \mathcal{F} \rightarrow X$ be the flag variety classifying flags

$$
0=F_{0} \subset F_{1} \subset \cdots \subset F_{m}=E
$$

such that the rank of $F_{i} / F_{i-1}$ is equal to $p_{i}$. Over $\mathcal{F}$ there is a universal flag $F^{u}$ on $p^{*} E$. Set $E_{i}:=F_{i}^{u} / F_{i-1}^{u}(i=1, \ldots m)$, so $E_{i}$ is a locally free sheaf of rank $p_{i}$ on $\mathcal{F}$.

Consider the polynomial ring $A_{\mathscr{M}}^{*, *}(X)\left[T_{i, j_{i}}\right]_{1 \leq i \leq m, 1 \leq j_{i} \leq p_{m}}$ with variable $T_{i, j_{i}}$ of bidegree $\left(j_{i}, j_{i}\right)$, and let $J$ be the bigraded ideal in this ring generated by the homogeneous elements (note that $c_{p}(E) \in A_{\mathscr{M}}^{p, p}(X)$ )

$$
c_{p}(E)-\sum_{i_{1}+\cdots+i_{m}=p} T_{1, i_{1}} \cdots T_{m, i_{m}}, \quad p \geq 1 .
$$

There is a map of bigraded rings

$$
\alpha: A_{\mathscr{M}}^{*, *}(X)\left[T_{i, j_{i}}\right] \rightarrow A_{\mathscr{M}}^{*, *}(\mathcal{F}), \quad T_{i, j_{i}} \mapsto c_{j_{i}}\left(E_{i}\right) .
$$

Proposition 3.6. The map $\alpha$ induces an isomorphism

$$
A_{\mathscr{M}}^{*, *}(X)\left[T_{i, j_{i}}\right] / J \simeq A_{\mathscr{M}}^{*, *}(\mathcal{F}) .
$$

Proof. This follows from the argument of [12, Exposé VII, §5]. Let us just indicate the necessary modifications here.

Let $f: D \rightarrow X$ be the flag variety classifying flags of type $(1,1, \ldots, 1)$ on $E$. There is a natural map $g: D \rightarrow \mathcal{F}$ realizing $D$ as the fiber product $D_{1} \times_{\mathcal{F}} D_{2} \cdots \times_{\mathcal{F}} D_{m}$, where $D_{i}$ is the variety over $\mathcal{F}$ classifying full flags in $E_{i}$.

Lemma 3.7. Let $S \in \mathscr{S}$ be an object and $E$ a locally free sheaf of rank $r$ on $S$ with associated projective bundle $p: \mathbb{P} E \rightarrow S$. Let $c_{1} \in H_{\mathscr{M}}^{2,1}(\mathbb{P} E)$ denote the first Chern class of the universal quotient, which induces a map $1_{S}(-1)[-2] \rightarrow p_{*} 1_{\mathbb{P E}}$. Then the map induced by summing the $\operatorname{maps} c_{1}^{i}$

in $\mathscr{M}(S)$ is an isomorphism.

$$
\oplus_{i=0}^{r-1} 1_{S}(-i)[-2 i] \rightarrow p_{*} 1_{\mathbb{P} E}
$$

Proof. Notice that we have $p_{\sharp} \simeq p_{!}(r-1)[2(r-1)] \simeq p_{*}(r-1)[2(r-1)]$, so the desired isomorphism can also be written as an isomorphism $M_{S}(\mathbb{P} E) \simeq \oplus_{i=0}^{r-1} 1_{S}(i)[2 i]$. This is shown in [6, Theorem 3.2].

Consider the algebra $A_{\mathscr{M}}^{*, *}(X)\left[U_{k}\right]_{k=1, \ldots, r}$ (with the $U_{k}$ of bidegree $(1,1)$ ) and the ideal $J_{D}$ generated by elements $c_{p}(E)-\sigma_{p}$, where $\sigma_{p}$ is the $p$-th symmetric function in the $U_{k}$. We then have a map

$$
\alpha_{D}: A_{\mathscr{M}}^{*, *}(X)\left[U_{k}\right] / J_{D} \rightarrow A_{\mathscr{M}}^{*, *}(D), \quad U_{k} \mapsto c_{1}\left(L_{k}\right)
$$

where $L_{k}$ is the $k$-th universal quotient on $D$. Factoring $f: D \rightarrow X$ as a sequence of projective bundles one sees that the map $\alpha_{D}$ is an isomorphism. Now let

$$
\theta: A_{\mathscr{M}}^{*, *}(X)\left[T_{i, j_{i}}\right] / J \rightarrow A_{\mathscr{M}}^{*, *}(X)\left[U_{k}\right] / J_{D}
$$

be the map induced by the map sending $T_{i, j_{i}}$ to the $j_{i}$-th elementary symmetric polynomial in the variables

$$
\left(U_{p_{1}+\cdots+p_{i-1}+s}\right)_{1 \leq s \leq p_{i}}
$$


We then have a commutative diagram

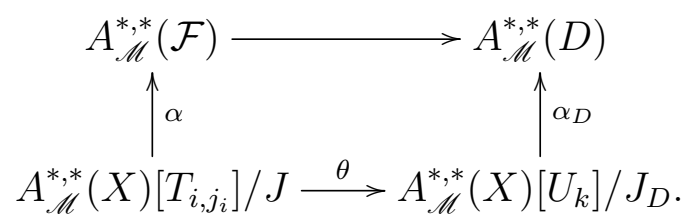

Analyzing this as in [12, p. 310] one gets that $\alpha$ is an isomorphism as well.

Remark 3.8. By the splitting principle and using 3.7, a theory of Chern classes is unique if it exists.

Corollary 3.9. Let $X \in \mathscr{S}$, let $E_{1}, \ldots, E_{s}$ be vector bundles on $X$, and let $v_{1}, \ldots, v_{s}$ be integers $\geq 0$. Let $G_{i}$ denote the Grassmanian of $v_{i}$-planes in $E_{i}$, and let $P_{i}$ denote the universal $v_{i}$-sub-bundle of $\left.E_{i}\right|_{G_{i}}$. Then the $A_{\mathscr{M}}^{* * *}(X)$-algebra $A_{\mathscr{M}}^{* * *}\left(\prod_{i} G_{i}\right)$ is generated by the homogeneous components of the elements $\operatorname{pr}_{i}^{*} c .\left(P^{i}\right)$.

Proof. This follows from the above description and factoring $\prod_{i} G_{i} \rightarrow X$ through a sequence of Grassman bundles.

\section{Local Chern Classes}

We continue with the notation of the preceding section.

4.1. For a closed imbedding $i: X \hookrightarrow M$ in $\mathscr{S}$, define

$$
A_{\mathscr{M}}^{n, m}(M \text { on } X):=\operatorname{Ext}_{\mathscr{M}(X)}^{2 n}\left(1_{X}, i^{!} 1_{M}(m)\right)
$$

and set

$$
A_{\mathscr{M}}^{*, *}(M \text { on } X):=\oplus_{n, m \in \mathbb{Z}} A_{\mathscr{M}}^{n, m}(M \text { on } X) .
$$

This is a bigraded module over $A_{\mathscr{M}}^{*, *}(X)$. A theory of local Chern classes consists of an as-

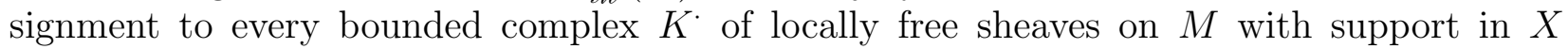
cohomology classes

$$
c_{i}^{M} \text { on } X\left(K^{\cdot}\right) \in A_{\mathscr{M}}^{i, i}(M \text { on } X)
$$

satisfying the following properties:

(i) (Pullback) If $f: M^{\prime} \rightarrow M$ is a morphism and $i^{\prime}: X^{\prime} \hookrightarrow M^{\prime}$ denotes $f^{-1}(X)$ then $f^{*} c_{i}^{M}$ on $X\left(K^{*}\right) \in A_{\mathscr{M}}^{i, i}\left(M^{\prime}\right.$ on $\left.X^{\prime}\right)$ is equal to $c_{i}^{M^{\prime}}$ on $X^{\prime}\left(f^{*} K^{*}\right)$.

(ii) Let $r: A_{\mathscr{M}}^{*, *}(M$ on $X) \rightarrow A_{\mathscr{M}}^{*, *}(M)$ be the map induced by the adjunction $i_{*} i^{!} \rightarrow$ id. Then if $c^{M}$ on $X\left(K^{*}\right) \in \prod_{i \geq 1} A_{\mathscr{M}}^{i, i}(M$ on $X)$ denotes the vector of the $c_{i}^{M}$ on $X\left(K^{*}\right)$ then

$$
r\left(c_{.}^{M} \text { on } X^{X}\left(K^{\cdot}\right)\right)+1=\prod_{i} c .\left(K^{2 i}\right) c .\left(K^{2 i-1}\right)^{-1}
$$

Using 3.6 and the argument of Iversen [14] one obtains:

Proposition 4.2. Suppose given a theory of Chern classes for $\mathscr{M}$. Then a theory of local Chern classes for $\mathscr{M}$ exists an is unique. 
4.3. In the case when $R$ is a $\mathbb{Q}$-algebra one can introduce as in [14, $\S 1]$ the localized Chern character

$$
\operatorname{ch}^{M} \text { on } X\left(K^{\cdot}\right) \in \prod_{i} A_{\mathscr{M}}^{i, i}(M \text { on } X) .
$$

By the argument of [14] this satisfies the following properties:

(i) (Functoriality) If $f: M^{\prime} \rightarrow M$ is a morphism and $i^{\prime}: X^{\prime} \hookrightarrow M^{\prime} \operatorname{denotes} f^{-1}(X)$ then $f^{*} \operatorname{ch}^{M}$ on $X\left(K^{\cdot}\right)=\operatorname{ch}^{M^{\prime}}$ on $X^{\prime}\left(f^{*} K^{\cdot}\right)$.

(ii) $r\left(\operatorname{ch}^{M}\right.$ on $\left.X\left(K^{\cdot}\right)\right)=\operatorname{ch}\left(K^{\cdot}\right)$.

(iii) (Decalage) $\mathrm{ch}^{M}$ on $X\left(K^{\cdot}[1]\right)=-\operatorname{ch}^{M}$ on $X\left(K^{\cdot}\right)$.

(iv) For complexes $K^{\cdot}$ and $L^{\cdot}$ on $M$ supported on $X$ we have

$$
\operatorname{ch}^{M} \text { on } X\left(K^{\cdot} \oplus L^{*}\right)=\operatorname{ch}^{M} \text { on } X\left(K^{*}\right)+\operatorname{ch}^{M} \text { on } X\left(L^{*}\right) \text {. }
$$

(v) (Multiplicativity) Let $K^{\cdot}$ (resp. $L^{\cdot}$ ) be a complex on $M$ supported on $Z$ (resp. $V$ ). Then

$$
\operatorname{ch}^{M} \text { on }(Z \cap V)\left(K^{*} \otimes L^{*}\right)=\operatorname{ch}^{M} \text { on } Z\left(K^{*}\right) \cdot \operatorname{ch}^{M} \text { on } V\left(L^{*}\right) .
$$

4.4. More generally, for a morphism $f: X \rightarrow Y$ in $\mathscr{S}$ we define $A_{\mathscr{M}}^{*, *}(f: X \rightarrow Y)$ by the formula

$$
A_{\mathscr{M}}^{n, m}(f: X \rightarrow Y):=\operatorname{Ext}_{\mathscr{M}(X)}^{n}\left(1_{X}, f^{!} 1_{Y}(m)\right) .
$$

Note that for a factorization of $f$

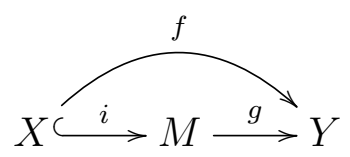

with $i$ an imbedding and $g$ smooth of relative dimension $d$ we have $f^{!} 1_{Y}(m) \simeq i^{!} g^{!} 1_{Y}(m) \simeq$ $i^{!} 1_{Y}(m+d)[2 d]$, whence a canonical isomorphism

$$
A_{\mathscr{M}}^{n, m}(f: X \rightarrow Y) \simeq A_{\mathscr{M}}^{n+d, m+d}(i: X \hookrightarrow M) .
$$

4.5. For a quasi-projective morphism $f: X \rightarrow Y$ in $\mathscr{S}$, one has the Grothendieck group of $f$-perfect complexes defined as in [16, 3.10]. Moreover, the same argument is in loc. cit. shows that there is a transformation

$$
\tau_{Y}^{X}: K(f \text {-perfect complexes on } X) \rightarrow \oplus_{i} A_{\mathscr{M}}^{i, i}(f: X \rightarrow Y) .
$$

This transformation is defined by choosing a factorization of $f$ as in 4.4.1 and sending a complex $K^{\cdot}$ to

$$
\operatorname{td}\left(i^{*} T_{M / Y}\right) \cdot \operatorname{ch}_{M}^{X}\left(K^{*}\right) \in A_{\mathscr{M}}^{*, *}(X \hookrightarrow M) \simeq A_{\mathscr{M}}^{*-d, *-d}(X \rightarrow Y)
$$

4.6. In particular, for $X \in \mathscr{S}$, quasi-projective over $B$, we can consider $A_{\mathscr{M}}^{n, m}(X \rightarrow B)$, with $X \rightarrow B$ the structure morphism. In this case we define

$$
H_{i, B M}^{\mathscr{M}}(X):=A_{\mathscr{M}}^{-i,-i}(X \rightarrow B),
$$

called the $i$-th $\mathscr{M}$-valued Borel-Moore homology of $X$ (or just $i$-th Borel-Moore homology if the reference to $\mathscr{M}$ is clear). In this case the Grothendieck group of $f$-perfect complexes is simply the Grothendieck group of coherent sheaves on $X$ (since $B$ is regular) so we get a map

$$
\tau_{X}: K(\operatorname{Coh}(X)) \rightarrow \oplus_{i} H_{i, B M}^{\mathscr{M}}(X)
$$


Remark 4.7. In the case when $B$ is the spectrum of a field, the map 4.6.1) can also be viewed as a cycle class map, using the identification of $K(\operatorname{Coh}(X))$ with Chow groups (tensor Q).

\section{LOCAL TERMS FOR MOTIVIC ACTIONS}

Let $k$ be a field and let $\mathscr{S}$ denote the category of finite type separated $k$-schemes. Fix a ring $R$ and let $\mathscr{M}$ be an $R$-linear triangulated motivic category.

5.1. Let $X, Y \in \mathscr{S}$ be two objects. For $F \in \mathscr{M}(X)$ and $G \in \mathscr{M}(Y)$ let $F \otimes G \in \mathscr{M}(X \times Y)$ denote $\operatorname{pr}_{1}^{*} F \otimes_{X \times Y} \operatorname{pr}_{2}^{*} G$. There is a map

$$
\epsilon_{X \times Y}: \Omega_{X} \otimes \Omega_{Y} \rightarrow \Omega_{X \times Y}
$$

defined as follows. We have isomorphisms

$$
\begin{array}{rlr}
\operatorname{Hom}_{X \times Y}\left(\Omega_{X} \otimes \Omega_{Y}, \Omega_{X \times Y}\right) & \simeq \quad \operatorname{Hom}_{X \times Y}\left(\operatorname{pr}_{1}^{*} \Omega_{X}, \operatorname{pr}_{2}^{!} 1_{Y}\right) \\
& \simeq & \operatorname{Hom}_{Y}\left(\operatorname{pr}_{2 !} \operatorname{pr}_{1}^{*} \Omega_{X}, 1_{Y}\right) \\
& \simeq \quad \operatorname{Hom}_{Y}\left(g^{*} f_{!} f^{!} 1_{\operatorname{Spec}(k)}, g^{*} 1_{\operatorname{Spec}(k)}\right),
\end{array}
$$

where $f: X \rightarrow \operatorname{Spec}(k)$ (resp. $g: Y \rightarrow \operatorname{Spec}(k))$ is the structure morphism, the first isomorphism is induced by the isomorphism $\operatorname{Hom}\left(\operatorname{pr}_{2}^{*} \Omega_{Y}, \Omega_{X \times Y}\right) \simeq \operatorname{pr}_{2}^{!} 1_{Y}$, the second isomorphism is by adjunction, and the third isomorphism is by base change. The map $\epsilon_{X \times Y}$ is the map corresponding under these isomorphisms to the adjunction map $f_{!} f^{!} 1_{\operatorname{Spec}(k)} \rightarrow 1_{\operatorname{Spec}(k)}$.

Assumption 5.2. Assume that the map $\epsilon_{X \times Y}$ is an isomorphism.

5.3. Note that there is a natural map

$$
1_{X} \otimes \Omega_{Y} \rightarrow \operatorname{Hom}_{X \times Y}\left(\Omega_{X} \otimes 1_{Y}, \Omega_{X} \otimes \Omega_{Y}\right)
$$

which, with the above identification of $\Omega_{X} \otimes \Omega_{Y}$ with $\Omega_{X \times Y}$, gives a map

$$
\rho_{X \times Y}: 1_{X} \otimes \Omega_{Y} \rightarrow D\left(\Omega_{X} \otimes 1_{Y}\right) .
$$

Lemma 5.4. The map $\rho_{X \times Y}$ is an isomorphism.

Proof. Consider a closed imbedding $i: Z \hookrightarrow Y$ with complement $j: U \hookrightarrow Y$, and let $\tilde{i}: X \times Z \hookrightarrow X \times Y$ and $\tilde{j}: X \times U \hookrightarrow X \times Y$ be the inclusions defined by base change. We then have a distinguished triangle (using assumption 5.2 )

$$
\tilde{i}_{*}\left(\Omega_{X} \otimes \Omega_{Z}\right) \rightarrow \Omega_{X} \otimes \Omega_{Y} \rightarrow \tilde{j}_{*}\left(\Omega_{X} \otimes \Omega_{U}\right) \rightarrow \tilde{i}_{*}\left(\Omega_{X} \otimes \Omega_{Z}\right)[1] .
$$

Applying $\operatorname{Hom}_{X \times Y}\left(\Omega_{X} \otimes 1_{Y},-\right)$ to this triangle we get a distinguished triangle

$$
\tilde{i}_{*} D\left(\Omega_{X} \otimes 1_{Z}\right) \rightarrow D\left(\Omega_{X} \otimes 1_{Y}\right) \rightarrow \tilde{j}_{*} D\left(\Omega_{X} \otimes 1_{U}\right) \rightarrow \tilde{i}_{*} D\left(\Omega_{X} \otimes 1_{Z}\right)[1] .
$$


Our map $\rho_{X \times Y}$ is compatible with this triangle, in the sense that we get a commutative diagram

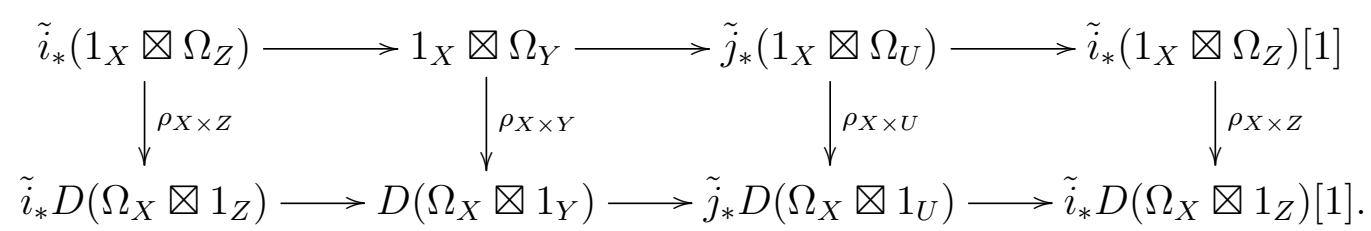

From this and induction on the dimension of $Y$ it follows that it suffices to consider the case when $Y$ is smooth of some dimension $d$, in which case the result is immediate.

5.5. Fix a correspondence $c: C \rightarrow X \times X$ with $C, X \in \mathscr{S}$ and fixed point scheme Fix $(c):=$ $C \times_{c, X \times X, \Delta_{X}} X$. For an action (a morphism in $\mathscr{M}(C)$ )

$$
u: c_{1}^{*} 1_{X} \simeq 1_{C} \rightarrow c_{2}^{!} 1_{X},
$$

we define $\operatorname{Tr}_{c}(u) \in H_{0, B M}^{\mathscr{M}}(\operatorname{Fix}(c)$ as follows. Note that we have

$$
c^{!}\left(\Omega_{X} \otimes 1_{X}\right) \simeq D_{C} c^{*}\left(1_{X} \otimes \Omega_{X}\right) \simeq D_{C} c_{2}^{*} \Omega_{X} \simeq c_{2}^{!} 1_{X} .
$$

Therefore we can also view $u$ as an element of $H_{\mathscr{M}}^{0}\left(C, c^{!}\left(\Omega_{X} \otimes 1_{X}\right)\right)$. Let $\Delta: X \rightarrow X \times X$ be the diagonal morphism and note that there is a natural map

$$
\Omega_{X} \otimes 1_{X} \rightarrow \Delta_{*} \Omega_{X}
$$

Applying this map and using the base change isomorphism for the cartesian diagram

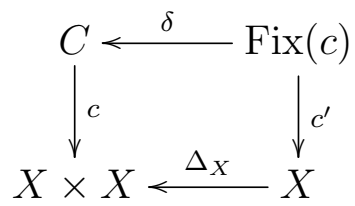

we get a morphism

$$
c^{!}\left(\Omega_{X} \otimes 1_{X}\right) \rightarrow c^{!} \Delta_{*} \Omega_{X} \simeq \delta_{*} c^{\prime !} \Omega_{X} \simeq \delta_{*} \Omega_{\mathrm{Fix}(c)}
$$

Applying $\operatorname{Hom}_{\mathscr{M}(C)}\left(1_{C},-\right)$ to this map we get a map

$$
\operatorname{Tr}^{\mathscr{M}}: \operatorname{Hom}_{\mathscr{M}(C)}\left(c_{1}^{*} 1_{X}, c_{2}^{!} 1_{X}\right) \rightarrow H_{0, B M}^{\mathscr{M}}(\operatorname{Fix}(c)) .
$$

If no confusion seems likely we write simply $\operatorname{Tr}$ for $\operatorname{Tr}^{\mathscr{M}}$.

5.6. Let $\mathscr{N}$ be a second motivic category satisfying the assumption 5.2 , and let

$$
R: \mathscr{M} \rightarrow \mathscr{N}
$$

be a morphism of fibered categories such that for every $X \in \mathscr{S}$ the morphism on fibers

$$
R_{X}: \mathscr{M}(X) \rightarrow \mathscr{N}(X)
$$

is a triangulated monoidal functor, and assume further that $R$ is compatible with the operations $f_{*}, f^{*}, f_{!}, f^{!}$for morphisms $f: X \rightarrow Y$ in $\mathscr{S}$, Tate twists, and internal Hom. Note that then $R$ induces for every $X \in \mathscr{S}$ a map (which we abusively also denote simply by $R$ )

$$
R: H_{*, B M}^{\mathscr{M}}(X) \rightarrow H_{*, B M}^{\mathscr{N}}(X) .
$$


Let $c: C \rightarrow X \times X$ be a correspondence as in 5.5, and let $u: c_{1}^{*} 1_{X, \mathscr{M}} \rightarrow c_{2}^{!} 1_{X, \mathscr{M}}$ be an action in $\mathscr{M}(C)$, where we write $1_{X, \mathscr{M}}$ for the unit object in $\mathscr{M}(X)$. Then it follows from the construction and the fact that $R$ is compatible with the six operations that

$$
R\left(\operatorname{Tr}^{\mathscr{M}}(u)\right)=\operatorname{Tr}^{\mathscr{N}}(R(u))
$$

in $H_{0, B M}^{\mathscr{N}}(\operatorname{Fix}(c))$.

\section{Beilinson motives}

In this section $B$ is a regular excellent scheme of finite Krull dimension $\delta$, and $\mathscr{S}$ denotes the category of finite type separated $B$-schemes.

6.1. For $R=\mathbb{Q}$, there are several equivalent constructions of triangulated motivic categories. The one most convenient for us in this paper is the category of constructible Beilinson motives defined in [4, §14] which we will denote by $\mathscr{M}_{B}$.

The main properties of this category that we will need are the following 6.2 and 6.5 (which in particular implies that 5.2 holds).

Proposition 6.2. For $X \in \mathscr{S}$ quasi-projective over $B$ the map 4.6.1 induces for every $i$ an isomorphism

$$
A_{\delta+i}(X)_{\mathbb{Q}} \simeq H_{i, B M}^{\mathscr{M}_{B}}(X)
$$

where the left side refers to Chow homology groups, tensor $\mathbb{Q}$, as defined in [11, §1.8] (or in the case when $B$ is the spectrum of a field [10, $\S 1.3])$.

Proof. To properly defined $\mathscr{M}_{B}$ requires a lot of preparatory material, for which we refer to [4, §14]. With notation as in [4, 14.1.2], we have a map of ring spectra

$$
H_{B} \rightarrow K G L_{\mathbb{Q}} \text {. }
$$

One definition of the category of Beilinson motives (see [4, 14.2.9]) is as the homotopy category $\mathrm{Ho}\left(H_{B}-\mathrm{mod}\right)$. The map 6.2.1 therefore induces a morphism of motivic categories

$$
\mathscr{M}_{B} \subset \operatorname{Ho}\left(H_{B}-\bmod \right) \rightarrow \operatorname{Ho}\left(K G L_{\mathbb{Q}}-\bmod \right) .
$$

Fix a closed imbedding $i: X \hookrightarrow M$, with $M$ smooth of constant dimension $d$ over $B$, and let $j: U \hookrightarrow M$ be the complement of $X$. Taking cohomology of the distinguished triangle

$$
i_{*} i^{!} 1_{M}(d+a)[2 d] \rightarrow 1_{M}(d+a)[2 d] \rightarrow j_{*} 1_{U}(d+a)[2 d]
$$

we get a long exact sequence

$$
\cdots \rightarrow H_{\mathscr{M}_{B}}^{s}\left(X, \Omega_{X}(a)\right) \rightarrow H_{\mathscr{M}_{B}}^{s+2 d, d+a}(M) \rightarrow H_{\mathscr{M}_{B}}^{s+2 d, d+a}(U) \rightarrow \cdots
$$

To compare this with K-theory we following the argument of [17, Proof of Théorème 8]. Recall from [17, 7.2 and Théoréme $8(\mathrm{v})]$ that for any $X \in \mathscr{S}$ the filtration $F$. on $K_{0}(X)_{\mathbb{Q}}$ defined by the Riemann-Roch isomorphism $K_{0}(X)_{\mathbb{Q}} \simeq A_{*}(X)_{\mathbb{Q}}\left(\right.$ so $\left.F_{j} / F_{j+1} \simeq A_{j}(X)_{\mathbb{Q}}\right)$ is induced by operations $\phi^{k}$ on $K_{0}(X)_{\mathbb{Q}}$. Following the proof of [17, Théorème 8 (ii)] we get the long exact sequence

$$
\cdots \rightarrow K_{m}(X) \rightarrow K_{m}(M) \rightarrow K_{m}(U) \rightarrow K_{m-1}(X) \rightarrow \cdots,
$$


and as in loc. cit. this sequence is compatible with the Adams operations and induces an exact sequence on the associated graded pieces

$$
\cdots \rightarrow \operatorname{gr}_{j} K_{m}(X) \rightarrow \operatorname{gr}_{j} K_{m}(M) \rightarrow \operatorname{gr}_{j} K_{m}(U) \rightarrow \cdots
$$

The fact that the map from K-theory to the cohomology of Beilinson motives is defined by the map of ring spectra 6.2.1 and the description of the Adam's operations as coming from the decomposition in [4, 14.1.1 (K5)] implies that we get an induced map from the long exact sequence 6.2 .3 to the long exact sequence 6.2.2. We therefore obtain a commutative diagram with exact rows

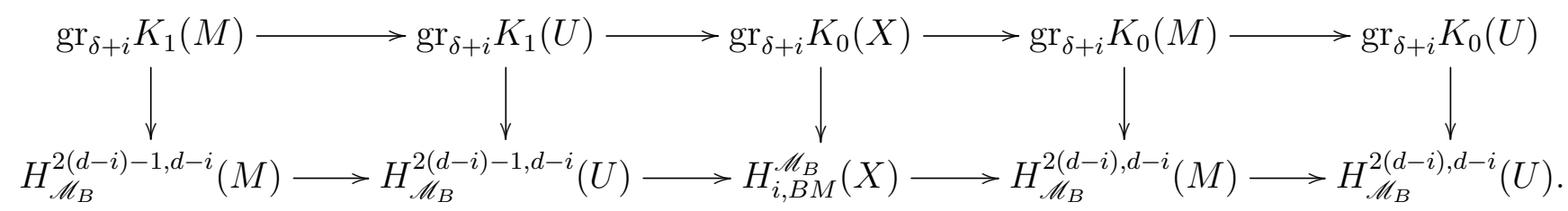

It therefore suffices to show that if $M \rightarrow B$ is smooth of relative dimension $d$ then for any integer $s \geq 0$ the map

$$
\operatorname{gr}_{\delta+i} K_{s}(M) \rightarrow H_{\mathscr{M}_{B}}^{2(d-i)-s, d-i}(M)
$$

is an isomorphism. This follows from [4, 14.2.14], which identifies $H_{\mathscr{M}_{B}}^{2(d-i)-s, d-i}(M)$ with $\operatorname{gr}_{\gamma}^{d-i} K_{s}(M)$ (associated graded of the $\gamma$-filtration), and [17, 7.2 (vi)] which shows that $\operatorname{gr}_{\gamma}^{d-i} K_{s}(M)=\operatorname{gr}_{i+\delta} K_{s}(M)$.

We therefore get an induced isomorphism $A_{i}(X)_{\mathbb{Q}} \simeq H_{i, B M}^{\mathscr{M}_{B}}(X)$. The fact that this isomorphism is induced by the map 4.6.1 follows from the uniqueness in 4.2

Remark 6.3. If $X \in \mathscr{S}$ is regular we have, by [4, 14.2.14], an isomorphism

$$
H_{\mathscr{M}_{B}}^{q, p}(X) \simeq \operatorname{gr}_{\gamma}^{p} K_{2 p-q}(X)_{\mathbb{Q}} .
$$

This isomorphism implies various vanishing results for motivic cohomology. We will need two cases in what follows:

(i) $(p<0)$ If $d$ is the dimension of $X$ then by [17, Théorème 7.2 (vi)] we have

$$
\operatorname{gr}_{\gamma}^{p} K_{2 p-q}(X)_{\mathbb{Q}}=\operatorname{gr}^{d-p} K_{2 p-q}(X)_{\mathbb{Q}},
$$

which in the notation of [17, 7.4] is equal to $H_{2 d-q}(X, d-p)$. If $p<0$ then $d-p>d$ in which case this group is 0 by [17, Théorème 8 (i)].

(ii) ( $X$ affine $p=0$ and $q<0$ ) In this case we have

$$
H_{\mathscr{M}_{B}}^{q, p}(X) \simeq \operatorname{gr}_{\gamma}^{0} K_{-q}(X)
$$

The vanishing of this group is a known special case of Beilinson-Soulé vanishing [17, $2.9]$.

The second property of the category of Beilinson motives that we will need is the following result, which will enable us to use de Jong's results on equivariant alterations [7].

6.4. Let $Y$ be a quasi-projective $k$-scheme and $G$ a finite group acting on $Y$. Let $X^{\prime}$ denote the coarse moduli space of the quotient stack $[Y / G]$ and let $\pi: X^{\prime} \rightarrow X$ be a finite surjective radicial morphism. Let $p: Y \rightarrow X$ be the projection and define $\left(p_{*} \Omega_{Y}\right)^{G}$ as in [4, 3.3.21]. 
There is a natural morphism $p_{*} \Omega_{Y} \rightarrow \Omega_{X}$ (dual to the adjunction morphism $1_{X} \rightarrow p_{*} 1_{Y}$ ) which induces a morphism

$$
h:\left(p_{*} \Omega_{Y}\right)^{G} \rightarrow \Omega_{X} .
$$

Proposition 6.5. The map 6.4.1 is an isomorphism.

Proof. Let $i: Z \hookrightarrow X$ be a closed imbedding with complement $j: U \hookrightarrow X$ such that the following hold:

(1) $U$ is everywhere dense in $X$.

(2) If $Y_{U}$ denotes $U \times_{X} Y$ then $U_{\text {red }}$ and $Y_{U \text {,red }}$ are regular, and the map $Y_{U \text {,red }} \rightarrow U_{\text {red }}$ is flat.

Let $Y_{Z}$ denote $Y \times_{X} Z$ and let $X_{Z}^{\prime}$ denote the coarse moduli space of $\left[Y_{Z} / G\right]$. The formation of the coarse moduli space does not in general commute with base change. It is still true, however, that $Y_{Z} \rightarrow X_{Z}^{\prime} \rightarrow Z$ satisfies the assumptions in 6.4. There is an induced map of distinguished triangles

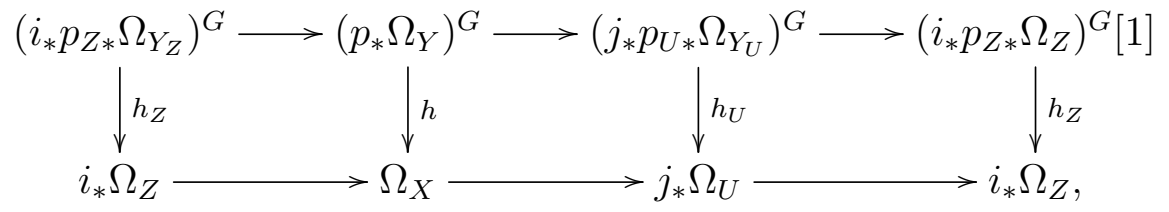

where we use the fact that the formation of homotopy fixed points commutes with pushforward. By induction we may assume that $h_{Z}$ is an isomorphism. It therefore suffices to consider the case when $X$ and $Y$ are regular of the same dimension, where it follows from [4, 3.3.35 and 14.3.3] and the following lemma.

Lemma 6.6. Let $X \in \mathscr{S}$ be regular of dimension $d$ and quasi-projective over $B$. Then $\Omega_{X} \simeq 1_{X}(d-\delta)[2(d-\delta)]$.

Proof. Since $X$ is quasi-projective over $B$ we can find a locally free sheaf $E$ of finite rank $r+1$ on $B$ and an imbedding $i: X \hookrightarrow \mathbb{P} E$ over $B$. Then $i$ is a regular imbedding of codimension $r+\delta-d$. We have $\Omega_{X} \simeq i^{!} 1_{\mathbb{P} E}(r)[2 r]$ so it suffices to show that $i^{!} 1_{\mathbb{P} E} \simeq 1_{X}(d-\delta-r)[2(d-\delta-r)]$. This follows from absolute purity for Beilinson motives [4, 14.4.1] (see also [5, A.2.8]).

An immediate corollary is the following:

Corollary 6.7. Let $Y \in \mathscr{S}$ be a regular quasi-projective $B$-scheme of dimension d, and let $G$ be a finite group acting on $Y$. Let $X:=Y / G$ be the coarse moduli space of the corresponding Deligne-Mumford stack $[Y / G]$. Then $\Omega_{X} \simeq 1_{X}(d-\delta)[2(d-\delta)]$.

In particular, if $f: Z \rightarrow X$ is a morphism then we have $A_{\mathscr{M}_{B}}^{i, i}(Z \rightarrow X) \simeq A_{i-d+\delta}(Z)_{\mathbb{Q}}$.

Proof. The first statement follows immediately from 6.5. The second statement follows from this and 6.2 .

6.8. For the remainder of this section we assume that $B$ is the spectrum of a field. In this case, proposition 6.5 also implies that assumption 5.2 holds for $\mathscr{M}_{B}$. The proof of this is a bit more intricate and it is useful to consider the following variant statements and intermediate results. 
6.9. Fix $X, Y \in \mathscr{S}$ and a closed imbedding $i: Z \hookrightarrow X$. Let $j: U \hookrightarrow X$ be the complement of $Z$ and let

$$
Z \times Y \stackrel{\tilde{i}}{\longrightarrow} X \times Y \stackrel{\tilde{j}}{\longleftarrow} U \times Y
$$

denote the base changes to $Y$. For $F \in \mathscr{M}_{B}(X)$ let $F_{U}$ denote the restriction to $U$, and consider the following conditions:

$\left(A_{X}^{Z}(F)\right)$ The natural map $\left(j_{*} F_{U}\right) \otimes \Omega_{Y} \rightarrow \tilde{j}_{*}\left(F_{U} \otimes \Omega_{Y}\right)$ is an isomorphism.

$\left(B_{X}^{Z}(F)\right)$ The natural map $\left(i^{!} F\right) \otimes \Omega_{Y} \rightarrow \tilde{i}^{!}\left(F \otimes \Omega_{Y}\right)$ is an isomorphism.

$\left(C_{X}\right)$ The map $\epsilon_{X \times Y}: \Omega_{X} \otimes \Omega_{Y} \rightarrow \Omega_{X \times Y}$ is an isomorphism.

Lemma 6.10. Properties $A_{X}^{Z}(F)$ and $B_{X}^{Z}(F)$ are equivalent.

Proof. Indeed the maps in question fit into a morphism of distinguished triangles

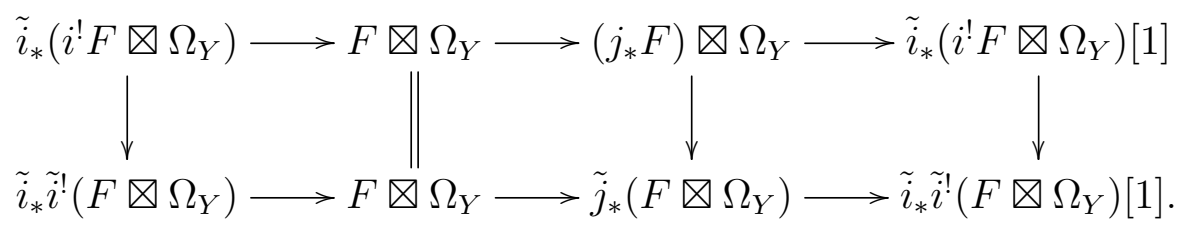

Lemma 6.11. Let $f: X^{\prime} \rightarrow X$ be a proper morphism, let $i^{\prime}: Z^{\prime} \hookrightarrow X^{\prime}$ be the preimage of $Z$, and let $F^{\prime} \in \mathscr{M}_{B}\left(X^{\prime}\right)$ be an object. Then $B_{X^{\prime}}^{Z^{\prime}}\left(F^{\prime}\right)$ implies $B_{X}^{Z}\left(f_{*} F^{\prime}\right)$.

Proof. Let $\tilde{f}: X^{\prime} \times Y \rightarrow X \times Y$ (resp. $f_{Z}: Z^{\prime} \rightarrow Z, \tilde{f}_{Z}: Z^{\prime} \times Y \rightarrow Z \times Y$ ) denote the morphism induced by $f$. Since $f$ is proper we have isomorphisms

$$
i^{!} f_{*} \simeq f_{Z *} i^{\prime !}, \quad \tilde{i} ! \tilde{f}_{*} \simeq \tilde{f}_{Z *} \tilde{i}^{\prime !} .
$$

From this and the projection formula for proper morphisms 2.8 (4) we get isomorphisms

$$
\left(i^{!} f_{*} F^{\prime}\right) \otimes \Omega_{Y} \simeq \tilde{f}_{Z *}\left(\left(i^{\prime !} F^{\prime}\right) \otimes \Omega_{Y}\right)
$$

and

$$
\tilde{i}^{!}\left(f_{*} F^{\prime} \otimes \Omega_{Y}\right) \simeq \tilde{f}_{Z *}\left(\tilde{i}^{\prime !}\left(F^{\prime} \otimes \Omega_{Y}\right)\right)
$$

which identifies the map in $B_{X}^{Z}\left(f_{*} F^{\prime}\right)$ with the pushforward of the map in $B_{X^{\prime}}^{Z^{\prime}}\left(F^{\prime}\right)$. From this the lemma follows.

Lemma 6.12. Let

$$
F_{1} \rightarrow F_{2} \rightarrow F_{3} \rightarrow F_{1}[1]
$$

be a distinguished triangle in $\mathscr{M}_{B}(X)$. If $B_{X}^{Z}\left(F_{1}\right)$ and $B_{X}^{Z}\left(F_{2}\right)$ hold then so does $B_{X}^{Z}\left(F_{3}\right)$.

Proof. This follows from noting that the morphisms in the properties $B_{X}^{Z}\left(F_{i}\right)$ fit into a morphism of distinguished triangles

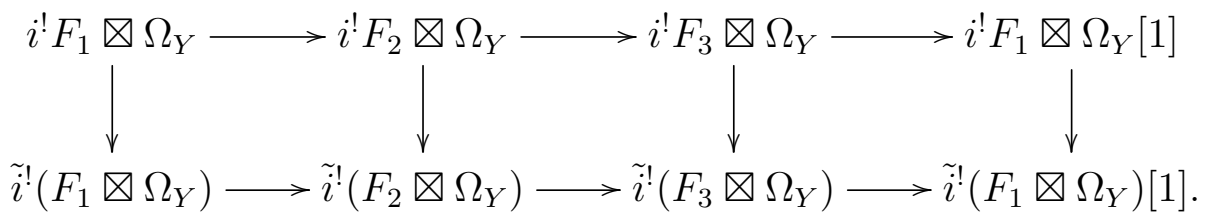


Lemma 6.13. Let $X \in \mathscr{S}$ be a scheme, and suppose that for every nowhere dense closed subscheme $i: Z \hookrightarrow X$ the properties $C_{Z}$ and $B_{X}^{Z}\left(\Omega_{X}\right)$ hold. Then property $C_{X}$ also holds.

Proof. Let $j: U \hookrightarrow X$ be an everywhere dense open subscheme with $U_{\text {red }}$ smooth over $k$, and let $i: Z \hookrightarrow X$ be the complementary closed subscheme (with the reduced structure). From the distinguished triangle

$$
i_{*} \Omega_{Z} \rightarrow \Omega_{X} \rightarrow j_{*} \Omega_{U} \rightarrow i_{*} \Omega_{Z}[1]
$$

and its variant for $X \times Y$ we get a commutative diagram

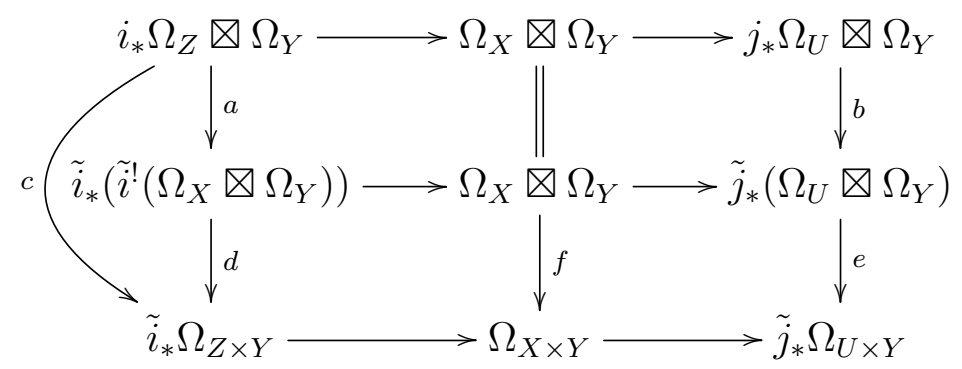

By property $B_{X}^{Z}\left(\Omega_{X}\right)$ the map labelled $a$ is an isomorphism. Since $U_{\text {red }}$ is smooth the map $e$ is trivially an isomorphism. Now by property $C_{Z}$ the map $c$ is an isomorphism whence the map $d$ is also an isomorphism. From this it follows that the map $f$ is an isomorphism as well.

Lemma 6.14. Let $i: Z \hookrightarrow X$ be a closed imbedding. The properties $C_{Z}$ and $C_{X}$ imply $B_{X}^{Z}\left(\Omega_{X}\right)$.

Proof. Indeed we have

$$
\left(i ! \Omega_{X}\right) \otimes \Omega_{Y} \simeq \Omega_{Z} \otimes \Omega_{Y} \simeq \Omega_{Z \times Y},
$$

where the second isomorphism is by property $C_{Z}$. Similarly we have

$$
\tilde{i} !\left(\Omega_{X} \otimes \Omega_{Y}\right) \simeq \tilde{i} !\left(\Omega_{X \times Y}\right) \simeq \Omega_{Z \times Y},
$$

where the first isomorphism is by property $C_{X}$. Under these identifications the map occurring in property $B_{X}^{Z}\left(\Omega_{X}\right)$ is identified with the identity map on $\Omega_{Z \times Y}$.

6.15. Let $p: E \rightarrow X$ be a proper morphism, and fix a distinguished triangle in $\mathscr{M}_{B}(X)$

$$
1_{X} \rightarrow p_{*} 1_{E} \rightarrow \mathcal{F} \rightarrow 1_{X}[1] .
$$

Assume there exists a closed imbedding $i: Z \hookrightarrow X$ with everywhere dense complement $j: U \rightarrow X$ such that the restriction $p_{U}: E_{U} \rightarrow U$ of $p$ to $U$ is finite radicial and surjective. Let $p_{Z}: E_{Z} \rightarrow Z$ be the restriction of $p$ to $Z$. Let $\mathcal{F}_{Z}$ denote a cone of the morphism $1_{Z} \rightarrow p_{Z *} 1_{E_{Z}}$ so we can find a morphism of distinguished triangles in $\mathscr{M}_{B}(X)$

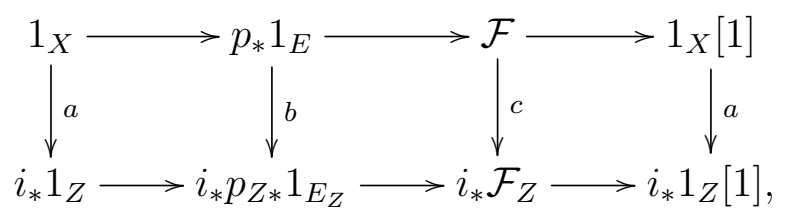

where the maps labelled $a$ and $b$ are the adjunction maps.

Lemma 6.16. The map $c: \mathcal{F} \rightarrow i_{*} \mathcal{F}_{Z}$ is an isomorphism. 
Proof. Considering the distinguished triangle

$$
j ! j^{*} \mathcal{F} \rightarrow \mathcal{F} \rightarrow i_{*} i^{*} \mathcal{F} \rightarrow j ! j^{*} \mathcal{F}[1]
$$

it suffices to show that $j^{*} \mathcal{F}=0$ and that the map $i^{*} \mathcal{F} \rightarrow \mathcal{F}_{Z}$ is an isomorphism. The first statement follows from the fact that the morphism $1_{X} \rightarrow p_{*} 1_{E}$ is an isomorphism over $U$, and the second statement follows from the fact that the base change map $i^{*} p_{*} 1_{E} \rightarrow p_{Z *} 1_{E_{Z}}$ is an isomorphism.

Theorem 6.17. Let $i: Z \hookrightarrow X$ be a closed imbedding in $\mathscr{S}$. Then properties $A_{X}^{Z}\left(1_{X}\right)$, $B_{X}^{Z}\left(1_{X}\right), A_{X}^{Z}\left(\Omega_{X}\right), B_{X}^{Z}\left(\Omega_{X}\right)$, and $C_{X}$ hold.

Proof. By induction on the dimension $d$ of $X$ we may assume that we have $X \in \mathscr{S}$ of dimension $d$ and that the theorem is true for every $E \in \mathscr{S}$ of dimension $<d$. To verify the theorem for $X$ it then suffices by 6.10 and 6.13 to show that for every $i: Z \hookrightarrow X$ the properties $B_{X}^{Z}\left(1_{X}\right)$ and $B_{X}^{Z}\left(\Omega_{X}\right)$ hold.

By [7, 7.3] we can find a proper morphism $\tilde{p}: \widetilde{E} \rightarrow X$ with $\widetilde{E}$ smooth and equipped with an action of finite group $G$ over $X$, such that if $p: E \rightarrow X$ is the coarse moduli space of the stack $[\widetilde{E} / G]$ then $p$ is generically on $X$ finite surjective and radicial. By 6.5 this implies that $\Omega_{E} \simeq 1_{E}(d)[2 d]$, where $d$ is the dimension of $\widetilde{E}$ (a locally constant function). This also implies that $\Omega_{E \times Y} \simeq 1_{E}(d)[2 d] \otimes \Omega_{Y}$ since by 6.5 we have an isomorphism

$$
\left(\tilde{p}_{*} \Omega_{\widetilde{E} \times Y}\right)^{G} \simeq\left(\tilde{p}_{*}\left(1_{\widetilde{E}}(d)[2 d] \otimes \Omega_{Y}\right)\right)^{G} \simeq\left(\tilde{p}_{*} \tilde{p}^{*}\left(1_{E}(d)[2 d] \otimes \Omega_{Y}\right)\right)^{G} \simeq 1_{E}(d)[2 d] \otimes \Omega_{Y},
$$

where the last isomorphism is by [4,3.3.35]. In particular, property $C_{E}$ holds.

This implies that for every closed $t: T \hookrightarrow E$ properties $B_{E}^{T}\left(\Omega_{E}\right)$ and $B_{E}^{T}\left(1_{E}\right)$ hold. Indeed since $E$ is obtained as the coarse moduli space of a smooth Deligne-Mumford stack, for any connected component $E_{i}$ of $E$ the intersection $T \cap E_{i}$ is either all of $E_{i}$ or of dimension $<d$. We can therefore apply 6.14 .

Let $Q$ be a cone of the morphism $1_{X} \rightarrow p_{*} 1_{E}$. By 6.11 we then have properties $B_{X}^{Z}\left(p_{*} 1_{E}\right)$ and $B_{X}^{Z}\left(p_{*} \Omega_{E}\right)$. Lemma 6.12 then implies that to verify property $B_{X}^{Z}\left(1_{X}\right)$ it suffices to verify the property $B_{X}^{Z}(Q)$. Dualizing we also have a distinguished triangle

$$
D_{X}(Q) \rightarrow p_{*} \Omega_{E} \rightarrow \Omega_{X} \rightarrow D_{X}(Q)[1]
$$

and to verify property $B_{X}^{Z}\left(\Omega_{X}\right)$ it suffices to verify property $B_{X}^{Z}\left(D_{X}(Q)\right)$.

Let $\alpha: T \hookrightarrow X$ be a nowhere dense closed subscheme such that the restriction of $p$ to the complement of $T$ is finite and radicial. Let $Z_{T}$ (resp. $\left.E_{T}, Z_{T}^{\prime}\right)$ denote $Z \cap T$ (resp. $T \times_{X} E$, $\left.Z_{T} \times_{X} E\right)$. Then by 6.16 we have $Q=\alpha_{*} Q_{T}$ for some $Q_{T} \in \mathscr{M}_{B}(T)$ fitting into a distinguished triangle

$$
1_{T} \rightarrow p_{T *} 1_{E_{T}} \rightarrow Q_{T} \rightarrow 1_{T}[1] .
$$

Dualizing we also get a distinguished triangle

$$
D_{T}\left(Q_{T}\right) \rightarrow p_{T *} \Omega_{E_{T}} \rightarrow \Omega_{T} \rightarrow D_{T}\left(Q_{T}\right)[1] .
$$

By the induction hypothesis and applying 6.12 we conclude that $B_{T}^{Z_{T}}\left(Q_{T}\right)$ and $B_{T}^{Z_{T}}\left(D_{T}\left(Q_{T}\right)\right)$ hold, and therefore by 6.11 properties $B_{X}^{Z}(Q)$ and $B_{X}^{Z}\left(D_{X}(Q)\right)$ also hold. 


\section{Application: local terms for aCtions given by lOCAlized Chern Classes}

Let $k$ be an algebraically closed field, and let $\mathscr{M}_{B}$ denote the motivic category of Beilinson motives over $k$.

7.1. For a prime $\ell$ invertible in $k$ there is constructed in [5, 5.9.21] an étale realization functor

$$
R_{\ell}: \mathscr{M}_{B} \rightarrow D M_{c, \ell}
$$

where for $X \in \mathscr{S}$ the fiber $D M_{c, \ell}(X)$ is isomorphic to the idempotent completion $D_{c}^{b}\left(X, \mathbb{Q}_{\ell}\right)^{\sharp}$ of the triangulated category $D_{c}^{b}\left(X, \mathbb{Q}_{\ell}\right)$. Here the idempotent completion is defined as in [2]. This realization functor is compatible with the six operations and Chern classes. Note also that by [2, 1.4] the functor

$$
D_{c}^{b}\left(X, \mathbb{Q}_{\ell}\right) \rightarrow D_{c}^{b}\left(X, \mathbb{Q}_{\ell}\right)^{\sharp}
$$

is fully faithful. Combining this with 7.2 we obtain the following:

Corollary 7.2. Let $c: C \rightarrow X \times X$ be a correspondence and let $u: c_{1}^{*} 1_{X} \rightarrow c_{2}^{!} 1_{X}$ be an action in $\mathscr{M}_{B}(C)$. Then there exists an algebraic cycle $\Sigma \in A_{0}(\operatorname{Fix}(c))_{\mathbb{Q}}$ such that for any prime $\ell$ invertible in $k$ we have $\operatorname{Tr}\left(u_{\ell}\right)=\operatorname{cl}(\Sigma)$, where $u_{\ell}: c_{1}^{*} \mathbb{Q}_{\ell} \rightarrow c_{2}^{!} \mathbb{Q}_{\ell}$ is the $\ell$-adic realization of $u$.

Proof. In fact the algebraic cycle $\Sigma$ is given by $\operatorname{Tr}^{\mathscr{M}_{B}}(u) \in H_{0, B M}^{\mathscr{M}_{B}}(\operatorname{Fix}(c)) \simeq A_{0}(\operatorname{Fix}(c))_{\mathbb{Q}}$.

7.3. We apply this to correspondences as follows. Let $c: C \rightarrow X \times X$ be a correspondence with $C$ and $X$ quasi-projective schemes, and let $E$ be a $c_{2}$-perfect complex on $C$. We then get an action $u_{\ell}: c_{1}^{*} \mathbb{Q}_{\ell} \rightarrow c_{2}^{!} \mathbb{Q}_{\ell}$ from the class $\tau_{X}^{C}(E) \in H^{0}\left(C, c_{2}^{!} \mathbb{Q}_{\ell}\right)$ defined in [16, 4.2].

Theorem 7.4. There exists a cycle $\Sigma \in A_{0}(\operatorname{Fix}(c))_{\mathbb{Q}}$, independent of $\ell$, such that $\operatorname{Tr}_{c}\left(u_{\ell}\right) \in$ $H^{0}\left(\operatorname{Fix}(c), \Omega_{\mathrm{Fix}(c)}\right)$ is equal to $\mathrm{cl}(\Sigma)$.

Proof. Since the $\ell$-adic realization functor is compatible with Chern classes 7.1, it is also compatible with localized Chern classes, by 4.2. Therefore the action $u_{\ell}$ is induced by a morphism in $\mathscr{M}_{B}(C)$ and the result follows from 7.2 .

\section{ApplicAtion: QUASI-FINITE MORPHiSMS AND CORRESPONDENCES}

In this section $B$ denotes a regular excellent scheme of dimension $\leq 2$, and $\mathscr{S}$ is the category of finite type separated $B$-schemes.

8.1. Let $\ell$ be a prime invertible on $B$, and let $f: Y \rightarrow X$ be a quasi-finite morphism between quasi-projective $B$-schemes. Let $u_{\ell} \in H^{0}\left(Y, f^{!} \mathbb{Q}_{\ell}\right)$ be a section. We say that $u_{\ell}$ is motivic if there exists a morphism $u: 1_{Y} \rightarrow f^{!} 1_{X}$ in $\mathscr{M}_{B}(Y)$ such that $u_{\ell}$ is the $\ell$-adic realization of $u$.

The condition that $u_{\ell}$ be motivic has the following more concrete characterization. Since $f$ is quasi-finite, $f_{!} \mathbb{Q}_{\ell}$ is a sheaf. For any dense open subscheme $j: U \hookrightarrow X$ the adjunction map $\mathbb{Q}_{\ell, X} \rightarrow R^{0} j_{*} \mathbb{Q}_{\ell, U}$ is injective, so the map $u_{\ell}$ is determined by its restriction to $f^{-1}(U)$. In particular, let $\left\{Y_{i}\right\}_{i \in I}$ be the irreducible components of $Y$ which dominate an irreducible component of $X$ via $f$, and choose a dense open subscheme $U \subset X$ such that $U_{\text {red }}$ is regular and

$$
f^{-1}(U)=\coprod_{i \in I} V_{i}
$$


where $V_{i} \subset Y_{i}$ is a dense open and $V_{i \text {,red }}$ is regular of the same dimension of its image in $U$. We then have a canonical isomorphism

$$
H^{0}\left(f^{-1}(U), f^{!} \mathbb{Q}_{\ell, U}\right) \simeq \mathbb{Q}_{\ell}^{I},
$$

and therefore we obtain an inclusion

$$
H^{0}\left(Y, f^{!} \mathbb{Q}_{\ell, X}\right) \hookrightarrow \mathbb{Q}_{\ell}^{I} .
$$

It follows immediately from the construction that this is independent of the choice of $U$. The image of $u_{\ell}$ in $\mathbb{Q}_{\ell}^{I}$ will be called the weight vector of $u_{\ell}$, and will be denoted $w\left(u_{\ell}\right)$.

Theorem 8.2. (i) The section $u_{\ell}$ is motivic if and only if the weight vector $w\left(u_{\ell}\right)$ lies in $\mathbb{Q}^{I} \subset \mathbb{Q}_{\ell}^{I}$.

(ii) If $u_{\ell}$ is the $\ell$-adic realization of $u: 1_{Y} \rightarrow f^{!} 1_{X}$, then for any other prime $\ell^{\prime}$ invertible in $k$ the $\ell^{\prime}$-adic realization $u_{\ell^{\prime}}$ of $u$ has $w\left(u_{\ell^{\prime}}\right)=w\left(u_{\ell}\right)$ in $\mathbb{Q}^{I}$.

Remark 8.3. If the weight vector $w\left(u_{\ell}\right)$ lies in $\mathbb{Q}^{I}$ we say that $u_{\ell}$ has rational weight vector.

The proof of 8.2 occupies the following $8.4-8.13$.

8.4. Fix a prime $\ell$ and an element $u_{\ell} \in H^{0}\left(Y, f^{!} \mathbb{Q}_{\ell}\right)$ with weight vector $w \in \mathbb{Q}^{I}$. We show that $u_{\ell}$ is motivic as follows.

8.5. By [8, 5.15] (in the case when $B$ is the spectrum of a field one can also use [7, 7.3]) we can find a proper morphism $\tilde{p}: \widetilde{E} \rightarrow X$ with $\widetilde{E}$ regular and equipped with an action of finite group $G$ over $X$, such that if $p: E \rightarrow X$ is the coarse moduli space of the stack $[\widetilde{E} / G]$ then $p$ is generically on $X$ finite surjective and radicial. Next choose a proper surjective generically finite morphism $\kappa: F \rightarrow Y \times_{f, X} E$, with $F$ regular, which fits into a commutative diagram

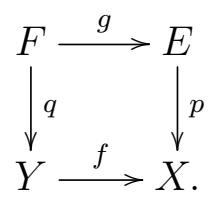

Let $\nu_{\ell}: \mathbb{Q}_{\ell} \rightarrow g^{!} \mathbb{Q}_{\ell}$ be the map which on a connected component $F$ dominating an irreducible component $Y_{i}$ of $Y$ is given by $w(u)_{i}$ divided by the number of irreducible components of $F$ which dominate $Y_{i}$. Here we are using the fact that since $E$ is the coarse moduli space of a regular Deligne-Mumford stack we have $\Omega_{E} \simeq \mathbb{Q}_{\ell}(d-\delta)[2(d-\delta)]$, where $d$ (resp. $\delta$ ) is the dimension of $E$ (resp. $B$ ), as in 6.7 .

Lemma 8.6. The diagram

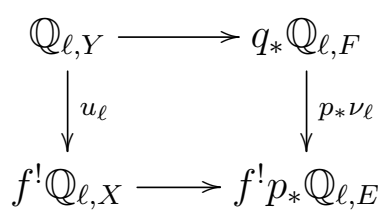

commutes.

Proof. It suffices to verify this at the generic point of each irreducible component of $X$, where the result is immediate from the construction. 
8.7. Fix a distinguished triangle in $\mathscr{M}_{B}(X)$

$$
1_{X} \rightarrow p_{*} 1_{E} \rightarrow \mathcal{F} \rightarrow 1_{X}[1]
$$

and let $\mathcal{F}_{\ell}$ denote the $\ell$-adic realization of $\mathcal{F}$, so we have a distinguished triangle in $D_{c}^{b}\left(X, \mathbb{Q}_{\ell}\right)$

$$
\mathbb{Q}_{\ell, X} \rightarrow p_{*} \mathbb{Q}_{\ell, E} \rightarrow \mathcal{F}_{\ell} \rightarrow \mathbb{Q}_{\ell, X}[1]
$$

Let $i: Z \hookrightarrow X$ be a closed imbedding with everywhere dense complement $j: U \rightarrow X$ such that the restriction $p_{U}: E_{U} \rightarrow U$ of $p$ to $U$ is finite radicial and surjective. Let $p_{Z}: E_{Z} \rightarrow Z$ be the restriction of $p$ to $Z$. Let $\mathcal{F}_{Z}$ denote a cone of the morphism $1_{Z} \rightarrow p_{Z *} 1_{E_{Z}}$ so we can find a morphism of distinguished triangles in $\mathscr{M}_{B}(X)$

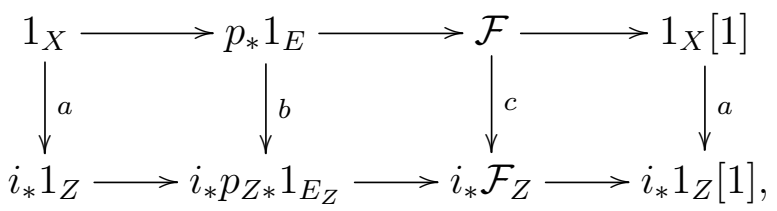

where the maps labelled $a$ and $b$ are the adjunction maps. By 6.16 the map $c: \mathcal{F} \rightarrow i_{*} \mathcal{F}_{Z}$ is an isomorphism.

Lemma 8.8. Let $f: Y \rightarrow X$ be a quasi-finite morphism of quasi-projective B-schemes. Then the $\ell$-adic realization map

$$
H_{\mathscr{M}_{B}}^{i}\left(Y, f^{!} 1_{X}\right) \rightarrow H^{i}\left(Y, f^{!} \mathbb{Q}_{\ell}\right)
$$

is injective for $i \leq 0$, and $H_{\mathscr{M}_{B}}^{i}\left(Y, f^{!} 1_{X}\right)=0$ for $i<0$.

Proof. The second statement follows from the first and the fact that the functor $f^{!}: D_{c}^{b}\left(X, \mathbb{Q}_{\ell}\right) \rightarrow$ $D_{c}^{b}\left(Y, \mathbb{Q}_{\ell}\right)$ takes $D_{c}^{\geq 0}\left(X, \mathbb{Q}_{\ell}\right)$ to $D_{c}^{\geq 0}\left(Y, \mathbb{Q}_{\ell}\right)$ by [1, XVIII, 3.1.7].

Consider first the case when $X$ is the coarse moduli space of a stack of the form $[M / G]$ with $M$ regular of some dimension $d$ and $G$ a finite group acting on $M$. In this case we have

$$
H_{\mathscr{M}_{B}}^{i}\left(Y, f^{!} 1_{X}\right) \simeq H_{\mathscr{M}_{B}}^{i}\left(Y, \Omega_{Y}(\delta-d)[2(\delta-d)]\right) .
$$

By 6.2 we therefore have

$$
H_{\mathscr{M}_{B}}^{0}\left(Y, f^{!} 1_{X}\right) \simeq H_{d-\delta, B M}^{\mathscr{M}}(Y) \simeq A_{d}(Y)_{\mathbb{Q}}
$$

Since $f$ is quasi-finite this is canonically isomorphic to the $\mathbb{Q}$-vector space with basis the irreducible components of $Y$ of dimension $d$. This implies the injectivity of 8.8 .1 for $i=0$, and also shows that if $j: V \subset Y$ is the preimage of a dense open subset in $X$ then the restriction map

$$
H_{\mathscr{M}_{B}}^{0}\left(Y, f^{!} 1_{X}\right) \rightarrow H_{\mathscr{M}_{B}}^{0}\left(V, f_{V}^{!} 1_{X}\right)
$$

is injective, where $f_{V}: V \rightarrow X$ is the restriction of $f$. Let $r: Z \hookrightarrow Y$ be the complement of $V$ and let $f_{Z}: Z \rightarrow X$ be the restriction of $f$. Choose $V$ such that $V_{\text {red }}$ is affine and regular of some dimension $e \leq d$. In this case we have $f_{V}^{!} 1_{X} \simeq 1_{V}(e-d)[2(e-d)]$ so

$$
H^{i}\left(V, f_{V}^{!} 1_{X}\right) \simeq H^{i+2(e-d)}\left(V, 1_{V}(e-d)\right) .
$$

By 6.3 these groups are zero if $i<0$. Now from the distinguished triangle

$$
r_{*} f_{Z}^{!} 1_{X} \rightarrow f^{!} 1_{X} \rightarrow j_{*} f_{V}^{!} 1_{X} \rightarrow r_{*} f_{Z}^{!} 1_{X}[1]
$$


we get a long exact sequence

$$
\cdots \rightarrow H^{i}\left(Z, f_{Z}^{!} 1_{X}\right) \rightarrow H^{i}\left(Y, f^{!} 1_{X}\right) \rightarrow H^{i}\left(V, f_{V}^{!} 1_{X}\right) \rightarrow \cdots
$$

By induction on the dimension of $Y$ (with base case handled by the case when $Y$ is smooth) we have $H^{i}\left(Z, f_{Z}^{!} 1_{X}\right)=0$ for $i<0$, and as discussed above we also have $H^{i}\left(V, f_{V}^{!} 1_{X}\right)=0$ for $i<0$. This therefore completes the proof in the case when $X$ is the coarse space of a stack $[M / G]$ as above.

For the general case we proceed by induction on the dimension of $X$. Let $p: E \rightarrow X$ be as in 8.5, and consider the resulting distinguished triangle

$$
1_{X} \rightarrow p_{*} 1_{E} \rightarrow \mathcal{F} \rightarrow 1_{X}[1]
$$

Applying $f^{!}$we get a distinguished triangle

$$
f^{!} 1_{X} \rightarrow f^{!} p_{*} 1_{E} \rightarrow f^{!} \mathcal{F} \rightarrow f^{!} 1_{X}[1] .
$$

Let $E_{Y}$ denote the fiber product $Y \times_{X} E$ so we have a cartesian square

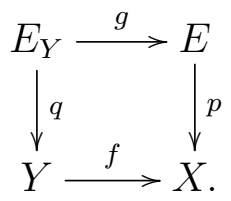

By base change, we have $f^{!} p_{*} 1_{E} \simeq q_{*} g^{!} 1_{E}$ and therefore

$$
H_{\mathscr{M}_{B}}^{i}\left(Y, f^{!} p_{*} 1_{E}\right) \simeq H_{\mathscr{M}_{B}}^{i}\left(E_{Y}, g^{!} 1_{E}\right) .
$$

By the regular case, it follows that the $\ell$-adic realization map

$$
H_{\mathscr{M}_{B}}^{i}\left(Y, f^{!} p_{*} 1_{E}\right) \rightarrow H^{i}\left(Y, f^{!} p_{*} \mathbb{Q}_{\ell}\right)
$$

is injective for $i \leq 0$. To prove the lemma it therefore suffices to show that $H^{i}(Y, \mathcal{F})=0$ for $i<0$. Let $i: Z \hookrightarrow X, Y_{Z}$ and $\mathcal{F}_{Z}$ be as in 8.7 so we have

$$
H^{i}\left(Y, f^{!} \mathcal{F}\right) \simeq H^{i}\left(Y_{Z}, f_{Z}^{!} \mathcal{F}_{Z}\right)
$$

Now consider the distinguished triangle on $Z$

$$
1_{Z} \rightarrow p_{Z *} 1_{E_{Z}} \rightarrow \mathcal{F}_{Z} \rightarrow 1_{Z}[1]
$$

and the resulting distinguished triangle

$$
f_{Z}^{!} 1_{Z} \rightarrow f_{Z}^{!} p_{Z *} 1_{E_{Z}} \rightarrow f_{Z}^{!} \mathcal{F}_{Z} \rightarrow f_{Z}^{!} 1_{Z}[1]
$$

on $Y_{Z}$. By induction the lemma holds for the quasi-finite morphisms $f_{Z}: Y_{Z} \rightarrow Z$ and $g_{Z}: E_{Y_{Z}} \rightarrow E_{Z}$. To prove that $H^{i}\left(Y_{Z}, f_{Z}^{!} \mathcal{F}_{Z}\right)=0$ for $i<0$ it therefore suffices to show that the map on étale cohomology

$$
H^{0}\left(Y_{Z}, f_{Z}^{!} \mathbb{Q}_{\ell}\right) \rightarrow H^{0}\left(E_{Y_{Z}}, g_{Z}^{!} \mathbb{Q}_{\ell}\right)
$$

is injective, which can be seen by restricting to a regular dense open subset.

8.9. Returning to the setting of 8.5 and 8.7 , fix also a distinguished triangle in $\mathscr{M}_{B}(Y)$

$$
1_{Y} \rightarrow q_{*} 1_{F} \rightarrow \mathcal{G} \rightarrow 1_{Y}[1]
$$

and let $\mathcal{G}_{\ell} \in D_{c}^{b}\left(Y, \mathbb{Q}_{\ell}\right)$ be the $\ell$-adic realization of $\mathcal{G}$. Let $\tilde{i}: Y_{Z} \hookrightarrow Y$ denote $f^{-1}(Z)$, let $q_{Z}: F_{Z} \rightarrow Y_{Z}$ denote the pullback of $q$, and let $\mathcal{G}_{Z}$ denote a cone of $1_{Y_{Z}} \rightarrow q_{Z *} 1_{F_{Z}}$. Then we have $\mathcal{G} \simeq \tilde{i}_{*} \mathcal{G}_{Z}$. 
8.10. Applying $i^{*}$ to the diagram 8.6.1 we obtain a commutative diagram

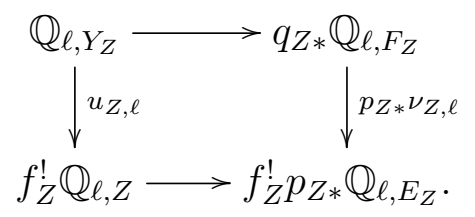

The map $\nu_{\ell}$ is motivic being given by the local Chern classes of a $g$-perfect complex of sheaves on $F$. The pullback $\nu_{Z}$ is therefore also motivic, whence the map $p_{Z *} \nu_{Z, \ell}$ is motivic as well.

Lemma 8.11. The weight vector of $u_{Z, \ell}$ is rational.

Proof. Let $W \subset Y_{Z}$ be an irreducible component which dominates an irreducible component $Z^{\prime} \subset Z$ via $f_{Z}$, and let $U^{\prime} \subset W$ be a nonempty regular open subset mapping to a regular open subset $U \subset Z^{\prime}$. Let $V^{\prime} \subset U^{\prime} \times_{Z} E_{Z}$ be a nonempty smooth open subset mapping to a smooth open subset $V \subset E_{Z}$. Note that since $U^{\prime}$ is quasi-finite over $Z, V^{\prime}$ is also quasi-finite over $E_{Z}$, whence $V^{\prime}$ and $V$ have the same dimension. Let $\alpha: U^{\prime} \rightarrow U$ (resp. $\beta: V^{\prime} \rightarrow V$ ) be the map induced by $f_{Z}\left(\operatorname{resp} . g_{Z}\right)$. Then $\alpha^{!} \simeq \alpha^{*}$. By the commutativity of 8.10 .1 , we then have a commutative diagram

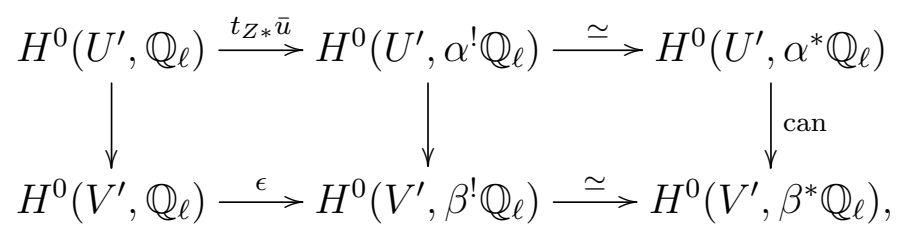

where if $d$ denotes the dimension of $V$ then $\epsilon$ is induced by an element of $A_{d}\left(V^{\prime}\right)_{\mathbb{Q}}=\mathbb{Q} \cdot\left[V^{\prime}\right]$ independent of $\ell$. It follows that the bottom horizontal composite in (8.11.1) is given by multiplication by a rational number independent of $\ell$, whence the top horizontal composite is also given by multiplication by a rational number independent of $\ell$.

8.12. By induction we can find $u_{Z}: 1_{Y_{Z}} \rightarrow f_{Z}^{!} 1_{Z}$ in $\mathscr{M}_{B}\left(Y_{Z}\right)$ with $\ell$-adic realization $u_{Z, \ell}$. Let $\nu_{Z}: 1_{F_{Z}} \rightarrow g_{Z}^{!} 1_{E_{Z}}$ denote a morphism in $\mathscr{M}_{B}\left(F_{Z}\right)$ inducing $\nu_{Z, \ell}$. By 8.8 the induced diagram

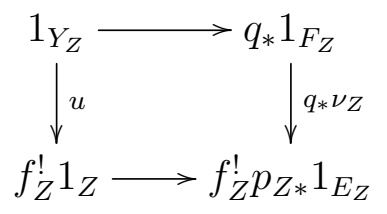

commutes since this holds for the $\ell$-adic realizations. Let $\rho_{Z}: \mathcal{G}_{Z} \rightarrow f_{Z}^{!} \mathcal{F}_{Z}$ be a morphism filling in the diagram

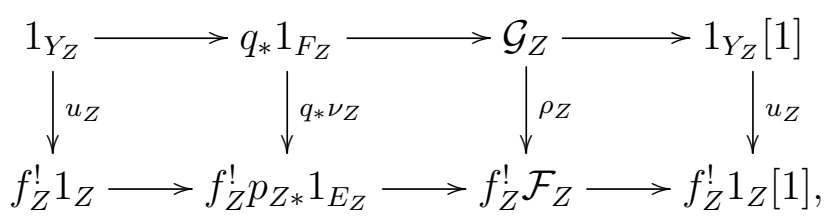


and let $\rho: \mathcal{G} \rightarrow f^{!} \mathcal{F}$ be the morphism obtained by applying $i_{*}$ to $\rho_{Z}$. Then the diagram

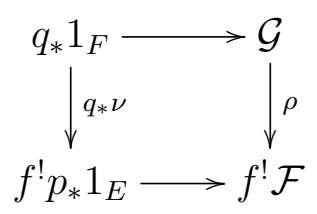

commutes. Indeed this can be verified after applying $i^{*}$ where the result follows from the construction. We can therefore find a morphism $\lambda: 1_{Y} \rightarrow f^{!} 1_{X}$ so that we have a morphism of distinguished triangles

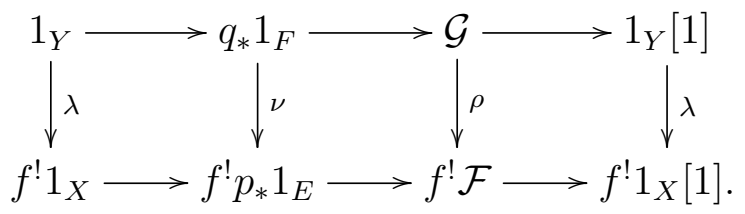

The $\ell$-adic realization of $\lambda$ is then equal to $u_{\ell}$, as this can be verified over a regular dense open of $X$. This completes the proof of the "if" part of statement (i).

8.13. To see the "only if" part of statement (i) as well as statement (ii) in 8.2 it suffices to define the weight vector of $u$ without passing to realizations. For this choose $U \subset X$ as in 8.15 so that $f^{-1}(U)=\coprod_{i} V_{i}$ with each $V_{i, \text { red }}$ regular. We then get a map

$$
H_{\mathscr{M}_{B}}^{0}\left(Y, f^{!} 1_{X}\right) \rightarrow \prod_{i} H_{\mathscr{M}_{B}}^{0}\left(V_{i}, f^{!} 1_{X}\right) \simeq \mathbb{Q}^{I}
$$

where the last isomorphism uses purity. The image of $u$ under this map is the weight vector. This completes the proof of 8.2 .

Remark 8.14. The proof (in particular 8.8) shows that if $u_{\ell}$ is motivic, then the morphism $u: 1_{Y} \rightarrow f^{!} 1_{X}$ in $\mathscr{M}_{B}(X)$ inducing $u_{\ell}$ is unique.

8.15. We apply this to correspondences as follows. Assume now that $B$ is the spectrum of an algebraically closed field $k$, and let $c: C \rightarrow X \times X$ be a correspondence with $X$ and $C$ quasi-projective schemes, and $c_{2}$ quasi-finite. Let $u: c_{1}^{*} \mathbb{Q}_{\ell} \rightarrow c_{2}^{!} \mathbb{Q}_{\ell}$ be an action of $c$ on $\mathbb{Q}_{\ell}$, which we can also view as a global section $u \in H^{0}\left(C, c_{2}^{!} \mathbb{Q}_{\ell, X}\right)$.

Theorem 8.16. Fix a weight vector $w \in \mathbb{Q}^{I}$, and assume that for some prime $\ell$ invertible in $k$ there exists an action $u_{\ell}: c_{1}^{*} \mathbb{Q}_{\ell} \rightarrow c_{2}^{!} \mathbb{Q}_{\ell}$ with $w(u)=w$.

(i) There exists an algebraic cycle $\Sigma \in A_{0}(\operatorname{Fix}(c))_{\mathbb{Q}}$ such that for any prime $\ell$ invertible in $k$ and action $u_{\ell}: c_{1}^{*} \mathbb{Q}_{\ell} \rightarrow c_{2}^{!} \mathbb{Q}_{\ell}$ with weight vector $w$ we have $\operatorname{Tr}_{c}\left(u_{\ell}\right)=\operatorname{cl}(\Sigma)$ in $H^{0}\left(\operatorname{Fix}(c), \Omega_{\mathrm{Fix}(c)}\right)$.

(ii) If $u$ has rational weight vector, then for every proper component $\Gamma \subset \operatorname{Fix}(c)$ the local term $\operatorname{lt}_{\Gamma}\left(\mathbb{Q}_{\ell, X}, u\right)$ is in $\mathbb{Q}$.

(iii) If $\ell$ and $\ell^{\prime}$ are two primes different from $p$, and $u \in H^{0}\left(C, c_{2}^{!} \mathbb{Q}_{\ell, X}\right)$ and $u^{\prime} \in H^{0}\left(C, c_{2}^{!} \mathbb{Q}_{\ell^{\prime}, X}\right)$ are sections with weight vectors $w(u)$ and $w\left(u^{\prime}\right)$ in $\mathbb{Q}^{I}$ and equal, then for every proper component $\Gamma \subset \operatorname{Fix}(c)$, we have equality of rational numbers

$$
\operatorname{lt}_{\Gamma}\left(\mathbb{Q}_{\ell}, u\right)=\operatorname{lt}_{\Gamma}\left(\mathbb{Q}_{\ell^{\prime}}, u^{\prime}\right)
$$


Proof. Statements (ii) and (iii) follow from (i). Statement (i) follows from 8.2 which implies that there exists a morphism $u: c_{1}^{*} 1_{X} \rightarrow c_{2}^{!} 1_{X}$ in $\mathscr{M}_{B}(C)$ such that for any prime $\ell$ invertible in $k$ the $\ell$-adic realization $u_{\ell}: c_{1}^{*} \mathbb{Q}_{\ell} \rightarrow c_{2}^{!} \mathbb{Q}_{\ell}$ of $u$ has weight vector $w$.

\subsection{Global consequences.}

Theorem 8.18. Let $c: C \rightarrow X \times X$ be a correspondence over the algebraic closure $k$ of a finite field with $C$ and $X$ Deligne-Mumford stacks, and assume $c_{2}$ is finite and $c_{1}$ is quasi-finite.

(i) If $u: c_{1}^{*} \mathbb{Q}_{\ell} \rightarrow c_{2}^{!} \mathbb{Q}_{\ell}$ is an action with rational weight vector $w(u)$, then $\operatorname{tr}\left(u \mid R \Gamma\left(X, \mathbb{Q}_{\ell}\right)\right)$ is in $\mathbb{Q}$.

(ii) If $\ell$ and $\ell^{\prime}$ are two primes and $u: c_{1}^{*} \mathbb{Q}_{\ell} \rightarrow c_{2}^{!} \mathbb{Q}_{\ell}$ and $u^{\prime}: c_{1}^{*} \mathbb{Q}_{\ell^{\prime}} \rightarrow c_{2}^{!} \mathbb{Q}_{\ell^{\prime}}$ are actions with rational weight vectors and $w(u)=w\left(u^{\prime}\right)$, then $\operatorname{tr}\left(u \mid R \Gamma\left(X, \mathbb{Q}_{\ell}\right)\right)=\operatorname{tr}\left(u^{\prime} \mid R \Gamma\left(X, \mathbb{Q}_{\ell^{\prime}}\right)\right)$.

Proof. Fix a model $c: C_{0} \rightarrow X_{0} \times X_{0}$ for $c$ over a finite field $\mathbb{F}_{q} \subset k$ such that all irreducible components of $C$ are defined over $\mathbb{F}_{q}$. Then any map $c_{1}^{*} \mathbb{Q}_{\ell} \rightarrow c_{2}^{!} \mathbb{Q}_{\ell}$ is defined over $\mathbb{F}_{q}$, and in particular commutes with Frobenius. For $n \geq 0$ let

$$
c^{(n)}: C \rightarrow X \times X
$$

be the correspondence given by $\left(c_{1}, F_{X}^{n} \circ c_{2}\right)$, where $F_{X}: X \rightarrow X$ is the base change to $k$ of the relative Frobenius on $X_{0}$.

If $u: c_{1}^{*} \mathbb{Q}_{\ell} \rightarrow c_{2}^{!} \mathbb{Q}_{\ell}$ is an action, let $u^{(n)}: c_{1}^{(n) *} \mathbb{Q}_{\ell} \rightarrow c_{2}^{(n) !} \mathbb{Q}_{\ell}$ be the action obtained by composing $u$ with the $n$ iterates of the canonical isomorphism $F_{X}^{!} \mathbb{Q}_{\ell} \rightarrow \mathbb{Q}_{\ell}$. Then as in $[13$, 3.5 (c)] to prove (i) it suffices to show that there exists $n_{0}$ such that for $n \geq n_{0}$ we have $\operatorname{tr}\left(u^{(n)} \mid R \Gamma\left(X, \mathbb{Q}_{\ell}\right)\right) \in \mathbb{Q}$ for all $n \geq n_{0}$, and to prove (ii) it suffices to show that there exists $n_{0}$ such that for all $n \geq n_{0}$ we have an equality of rational numbers

$$
\operatorname{tr}\left(u^{(n)} \mid R \Gamma\left(X, \mathbb{Q}_{\ell}\right)\right)=\operatorname{tr}\left(u^{\prime(n)} \mid R \Gamma\left(X, \mathbb{Q}_{\ell^{\prime}}\right)\right) .
$$

Let $d: C \rightarrow X \times X$ be the transpose of $c$ given by $\left(c_{2}, c_{1}\right)$. For $n \geq 0$ let ${ }^{(n)} d: C \rightarrow X \times X$ denote the correspondence $\left(F_{X}^{n} c_{2}, c_{1}\right)$, so ${ }^{(n)} d$ is the transpose of $c^{(n)}$. Let $v: d_{1}^{*} \Omega_{X} \rightarrow d_{2}^{!} \Omega_{X}$ denote the transpose of $u$, and for $n \geq n_{0}$ let ${ }^{(n)} v:{ }^{(n)} d_{1}^{*} \Omega_{X} \rightarrow{ }^{(n)} d_{2}^{!} \Omega_{X}$ denote the map obtained by $n$ iterates of the isomorphism $F_{X}^{*} \Omega_{X} \rightarrow \Omega_{X}$.

By Fujiwara's theorem [9, 5.4.5] there exists an integer $n_{0}$ such that for all $n \geq n_{0}$ the following hold:

(i) The fixed points $\operatorname{Fix}\left({ }^{(n)} d\right)=\operatorname{Fix}\left(c^{(n)}\right)$ consists of a finite set of isolated points.

(ii) We have

$$
\operatorname{tr}\left({ }^{(n)} v \mid R \Gamma_{c}\left(X, \Omega_{X}\right)\right)=\sum_{y \in \operatorname{Fix}((n) d)} \operatorname{lt}_{y}\left(\Omega_{X},{ }^{(n)} v\right) .
$$

(iii) If $U \rightarrow X$ is an étale morphism and $d_{U}: C_{U} \rightarrow U \times U$ denotes the pullback of $c$ along $U \times U \rightarrow X \times X$, and if $v_{U}: d_{U 1}^{*} \Omega_{U} \rightarrow d_{U 2}^{!} \Omega_{U}$ denotes the pullback of $v$, then for every $y^{\prime} \in \operatorname{Fix}\left({ }^{(n)} d_{U}\right)=U \times_{X} \operatorname{Fix}\left({ }^{(n)} d\right)$ mapping to $y \in \operatorname{Fix}\left({ }^{(n)} d\right)$ we have

$$
\operatorname{lt}_{y^{\prime}}\left(\Omega_{U},{ }^{(n)} d_{U}\right)=\operatorname{lt}_{y}\left(\Omega_{X},{ }^{(n)} d\right) .
$$

Now since ${ }^{(n)} v$ is adjoint to $u^{(n)}$ we have

$$
\operatorname{tr}\left({ }^{(n)} v \mid R \Gamma_{c}\left(X, \Omega_{X}\right)\right)=\operatorname{tr}\left(u^{(n)} \mid R \Gamma\left(X, \mathbb{Q}_{\ell}\right)\right),
$$


and by [12, III, 5.1.6] we have

$$
\operatorname{lt}_{y}\left(\Omega_{X},{ }^{(n)} v\right)=\operatorname{lt}_{y}\left(\mathbb{Q}_{\ell}, u^{(n)}\right) .
$$

It follows that for $n \geq n_{0}$

$$
\operatorname{tr}\left(u^{(n)} \mid R \Gamma\left(X, \mathbb{Q}_{\ell}\right)\right)=\sum_{y \in \operatorname{Fix}\left(c^{(n)}\right)} \operatorname{lt}_{y}\left(\mathbb{Q}_{\ell}, u^{(n)}\right) .
$$

Now by (iii), the local term $\operatorname{lt}_{y}\left(\mathbb{Q}_{\ell}, u^{(n)}\right)$ can be computed after replacing $X$ by an étale covering, which reduces the computation to the case when $X$ is quasi-projective. Combining this with 8.16 we get the theorem (note that if $w(u)_{i}$ denotes the component of the weight vector corresponding to an irreducible component $C_{i} \subset C$ then $\left.q^{\operatorname{dim}\left(C_{i}\right)} w(u)_{i}=w\left(u^{(n)}\right)_{i}\right)_{\text {. }}$

\section{REFERENCES}

[1] M. Artin, A. Grothendieck, and J.-L. Verdier, Théorie des topos et cohomologie étale des schémas, Springer Lecture Notes in Mathematics 269, 270, 305, Springer-Verlag, Berlin (1972).

[2] P. Balmer and M. Schlichting, Idempotent completion of triangulated categories, J. of Algebra 236 (2001), 819-834.

[3] M. Bondarko, Differential graded motives: weight complex, weight filtrations and spectral sequences for realizations; Voevodsky vs. Hanamura, preprint available on arXiv:math/0601713 (2008).

[4] D.-C. Cisinski and F. Déglise, Triangulated categories of mixed motives, preprint (2012).

[5] D.-C. Cisinski and F. Déglise, Étale Motives, preprint (2013).

[6] F. Déglise, Around the Gysin triangle II, Doc. Math. 13 (2008), 613-675.

[7] A.J. de Jong, Smoothness, semi-stability and alterations, Inst. Hautes Études Sci. Publ. Math. 83 (1996), 51-93.

[8] A. J. de Jong, Families of curves and alterations, Ann. Inst. Fourier 47 (1997). 599-621.

[9] K. Fujiwara, Rigid geometry, Lefschetz-Verdier trace formula, and Deligne's conjecture, Inv. Math. 127, 489-533 (1997).

[10] W. Fulton, Intersection Theory, Second Edition, Ergebnisse der Mathematik, Springer-Verlag, Berlin (1998).

[11] W. Fulton, Rational equivalence on singular varieties, Publ. Math. IHES 45 (1975), 147-167.

[12] A. Grothendieck, Cohomologie l-adic et Fonctions L, Lectures Notes in Math 589 (1977).

[13] L. Illusie, Miscellany on traces in $\ell$-adic cohomology: a survey, Jpn. J. Math 1 (2006), 107-136.

[14] B. Iversen, Local Chern classes, Ann. Sci. École Norm. Sup. 9 (1976), 155-169.

[15] H. Krause, On Neeman's well generated triangulated categories, Doc. Math. 6 (2001), 121-126.

[16] M. Olsson, Borel-Moore homology, intersection products, and local terms, preprint (2014).

[17] C. Soulé, Opérations en K-théorie algébrique, Canad. J. Math. 37 (1985), 488-550.

[18] Y. Varshavsky, Lefschetz-Verdier trace formula and a generalization of a theorem of Fujiwara, Geom. Funct. Anal. 17 (2007), 271-319. 